\title{
Connecting a Set of Circles with Minimum Sum of Radii
}

\author{
Erin W. Chambers ${ }^{\mathrm{a}}$, Sándor P. Fekete ${ }^{\mathrm{b}, *}$, Hella-Franziska Hoffmann ${ }^{\mathrm{c}}$, Dimitri Marinakis ${ }^{\mathrm{d}}$, \\ Joseph S. B. Mitchelle, Venkatesh Srinivasan ${ }^{\mathrm{f}}$, Ulrike Stege ${ }^{\mathrm{f}}$, Sue Whitesides ${ }^{\mathrm{f}}$ \\ ${ }^{a}$ Department of Computer Science, Saint Louis University, USA \\ ${ }^{b}$ Department of Computer Science, TU Braunschweig, Germany \\ ${ }^{c}$ David R. Cheriton School of Computer Science, University of Waterloo, Canada \\ ${ }^{d}$ Kinsol Research Inc., Duncan, BC, Canada \\ ${ }^{e}$ Department of Applied Mathematics and Statistics, Stony Brook University, USA \\ ${ }^{f}$ Department of Computer Science, University of Victoria, Canada
}

\begin{abstract}
We consider the problem of assigning radii to a given set of points in the plane, such that the resulting set of disks is connected, and the sum of radii is minimized. We prove that the problem is NP-hard in planar weighted graphs if there are upper bounds on the radii and sketch a similar proof for planar point sets. For the case when there are no upper bounds on the radii, the complexity is open; we give a polynomial-time approximation scheme. We also give constant-factor approximation guarantees for solutions with a bounded number of disks; these results are supported by lower bounds, which are shown to be tight in some of the cases. Finally, we show that the problem is polynomially solvable if a connectivity tree is given, and we conclude with some experimental results.
\end{abstract}

Keywords: Intersection graphs, connectivity problems, NP-hardness problems, approximation, upper and lower bounds.

\section{Introduction}

Problems of connectivity are among the most fundamental ones for many types of networks. Typically, these arise in a geometric setting, e.g., when considering the relation between the location of transmitters, the range of their transmissions, and their ability to connect. As a result, important aspects include the underlying geometry, the nature of connectivity, and the study of corresponding cost functions. Thus, connectivity problems bring together graph theory with computational geometry, a combination that was always dear to Ferran Hurtado; e.g., see [1] for a study of connectivity that mixes graphs and geometry.

In this paper, we consider a natural connectivity problem, with a focus on the geometric aspects, arising from assigning ranges to a set of points, such that the resulting disk intersection graph is connected. More precisely, we are given a set of points $P=\left\{p_{1}, \ldots, p_{n}\right\}$ in the plane. Each point $p_{i}$ is assigned a range $r_{i}$, inducing a disk of radius $r_{i}$. Two points $p_{i}, p_{j}$ are adjacent in the connectivity graph $H$, if their disks intersect. The Connected Range Assignment Problem (CRA) requires an assignment of radii to $P$, such that the objective function $R=\sum_{i} r_{i}^{\alpha}, \alpha=1$ is minimized, subject to the constraint that $H$ is connected.

Problems of this type have been considered before and have natural motivations from fields including networks, robotics, and data analysis, where ranges have to be assigned to a set of devices, and the total cost is given by an objective function that considers the sum of the radii of disks to some exponent $\alpha$. The cases $\alpha=2$ or 3 correspond to minimizing the overall power. The motivation for the case $\alpha=1$ arises from

\footnotetext{
${ }^{*}$ Corresponding author

Email addresses: echambe5@slu.edu (Erin W. Chambers), s.fekete@tu-bs.de (Sándor P. Fekete), hrhoffma@uwaterloo.ca (Hella-Franziska Hoffmann), dmarinak@kinsolresearch.com (Dimitri Marinakis), jsbm@ams.sunysb.edu (Joseph S. B. Mitchell), srinivas@uvic.ca (Venkatesh Srinivasan), ustege@uvic.ca (Ulrike Stege), sue@uvic.ca (Sue Whitesides)
} 
scanning the corresponding ranges with a minimum required angular resolution, so that the scan time for each disk corresponds to its perimeter, and thus radius.

\subsection{Related Work}

There is a large body of literature on algorithmic methods for range assignment in wireless sensor and ad-hoc networks; see Calinescu et al. 6, Calinescu and Wan [7, Carmi and Katz 9, Carmi et al. 11, Caragiannis et al. [8, Lloyd et al. 20, Wan et al. 23] for a (small) selection of aspects and variants.

In the context of clustering, Doddi et al. 15, Charikar and Panigraphy [13, and Gibson et al. 17] consider the following problems. Given a set $P$ of $n$ points in a metric space with metric $d(i, j)$ and an integer $k$, partition $P$ into a set of at most $k$ clusters with minimum sum of either (a) cluster diameters, or (b) cluster radii. Thus, the most significant difference with our problem is the lack of a connectivity constraint. Doddi et al. [15] provide approximation results for (a). They present a polynomial-time algorithm, which returns $O(k)$ clusters that are $O\left(\log \left(\frac{n}{k}\right)\right)$-approximate. For a fixed $k$, they transform an instance of their problem into a min-cost set-cover problem instance yielding a polynomial-time 2-approximation. They also show that the existence of a $(2-\epsilon)$-approximation would imply $P=N P$. In addition, they prove that the problem in weighted graphs without triangle inequality cannot be efficiently approximated within any factor, unless $P=N P$. Note that every solution to (b) is a 2-approximation for (a). Thus, the approximation results can be applied to case (a) as well. A greedy logarithmic approximation and a primal-dual based constant factor approximation for minimum sum of cluster radii is provided by Charikar and Panigraphy [13.

Another often considered setting is the one in which the coverage of a given set of base stations is required. In this setting, Alt et al. 2] consider a closely related problem of selecting disk centers and radii such that a given set of points in the plane are covered by the disks. Like our work, they focus on minimizing an objective function based on $\sum_{i} r_{i}^{\alpha}$ and produce results specific to various values of $\alpha$. The minimum sum of radii disk coverage problem (with $\alpha=1$ ) is also considered by Lev-Tov and Peleg [18] in the context of radio networks. Again, connectivity is not a requirement. In a more geometric setting, Bilò et al. [5] provide approximation schemes for the minimum size $k$-clustering problem that requires dividing the set of centers into at most $k$ clusters with minimum cluster cost.

A lot of work has also been done on radii/range assignment problems which require the special connectivity of "communication". The work of Clementi et al. [14] considers minimal assignments of transmission power to devices in a wireless network such that the network stays connected. In that context, the objective function typically considers an $\alpha>1$ based on models of radio wave propagation. Furthermore, in the type of problem considered by Clementi et al. the connectivity graph is directed; i.e. the power assigned to a specific device affects its transmission range, but not its reception range. This is in contrast to our work in which we consider an undirected connectivity graph. See Fuchs [16] for a collection of hardness results of different (directed) communication graphs. Carmi et al. [10] prove that an Euclidean minimum spanning tree is a constant-factor approximation for a variety of problems including the Minimum-Area Connected Disk Graph problem, which equals our problem with the different objective of minimizing the area of the union of disks, while we consider minimizing the sum of the radii (or perimeters) of all disks. This can apply to a hybrid robot or sensor network system such as that described in Marinakis et al. 21]: Consider a situation in which a mobile robot is required to visit the region of each static network component, e.g., for environmental monitoring purposes, and requires constant one-way information from at least one static network component at all times; e.g., for navigational purposes. In this case, a reasonable objective is to reduce the area covered by the network while maintaining connectivity.

\subsection{Our Work}

In this paper we investigate a variety of algorithmic aspects of the CRA problem. In Section 2 , we show that for a given connectivity tree, an optimal solution can be computed efficiently. Section 3 gives a proof of NP-hardness for the problem when there is an upper bound on the radii, with full details for the case of planar weighted graphs and a sketch for planar point sets. Section 4 provides a number of approximation results for solutions with bounded number of disks. In Section 5, we present a PTAS for the case in which there are no upper bounds on the radii of the disks. These theoretical results are complemented by experimental results in Section 6. A concluding discussion with open problems is provided in Section 7. 


\section{CRA for a Given Connectivity Tree}

In some settings, a specific set of connectivity edges is required instead of any connectivity tree. In this section, we will show that the Connected Range Assignment problem is solvable in polynomial time, when we are given a specific connectivity tree. First we give a formal definition of this variant.

In the Connected Range Assignment Problem for a Given Connectivity Tree (CRACT), we are given a finite set $P$, a metric $d$ and a spanning tree $T$ for the vertex set $P$. We are required to find an assignment $r_{i}$ of radii for point $p_{i}$ in $P$ such that $R=\sum_{i} r_{i}$ is minimized subject to the constraint that if $\left(p_{i}, p_{j}\right)$ is an edge in $T$, then the two corresponding disks $C_{i}$ and $C_{j}$ (with radii $r_{i}$ and $r_{j}$ respectively) intersect.

We call a solution for Problem CRACT an optimal range assignment for $T$. We first state some structural properties of optimal range assignments and then, based on those, provide a polynomial-time algorithm for Problem CRACT. In the following, we always consider the case where $|P|$ is at least 2 and assume that $T$ is rooted at some internal node $p_{r}$ of $T$.

Lemma 1. Given a connectivity tree $T$ with at least three nodes, there exists an optimal range assignment for $T$ with $r_{l}=0$ for all leaves $p_{l}$ of $T$.

Proof. Consider a CRACT instance $(P, d, T)$. Assume an optimal range assignment $r$ for $T$ has a leaf $p_{l} \in P$ with $r_{l}>0$. Then, the disk $C_{l}$ around $p_{l}$ with radius $r_{l}$ must intersect the disk $C_{u}$ around $p_{l}$ 's parent $p_{u}$ with radius $r_{u}$. We extend $r_{u}$ to $r_{u}:=r_{u}+r_{l}$ and set $r_{l}:=0$. This does not increase $R=\sum_{p_{i} \in P} r_{i}$ and maintains a solution for $T$.

The following lemma extends Lemma 1. It shows that there is an optimal range assignment for which it is possible to compute the radius assigned to any point $p$ of height $h$, given the radius assigned to its children.

Lemma 2. Given a CRACT instance $(P, d, T)$, there is an optimal range assignment $r$ for $T$ that satisfies the following conditions:

1. $r_{l}=0$ for all leaves $p_{l}$ of $T$

2. For any node $p_{u} \in P$ of height $h, 1 \leq h \leq h_{T}$, in $T$,

$$
r_{u}=\max _{p_{c} \text { is child of } p_{u}} d\left(p_{c}, p_{u}\right)-r_{c}
$$

Proof. To prove that $r$ satisfying the two conditions above is an optimal range assignment, we show that any optimal range assignment can be gradually converted into assignment $r$ in a sequence of $h_{T}+1$ steps as follows: At the end of step $i$, all nodes $p_{u}$ in $T$ with height $h, 0 \leq h \leq i-1$, will be assigned the radius $r_{u}$. Furthermore, the sum of radii at the end of step $i$ is at most the sum of radii at the end of step $i-1$.

[Step 1] Perform the transformation as described in the proof of Lemma 1. It is easily seen that the condition (1) is true at the end of step 1.

[Step $i$ ] Let $p_{u}$ be a node of height $i-1$ in $T$. Further let $d_{\max }\left(p_{u}\right):=\max d\left(p_{c}, p_{u}\right)$ over all children $p_{c}$ of $p_{u}$. Then we have $r_{u} \geq d_{\max }\left(p_{u}\right)$. If $r_{u}>d_{\max }\left(p_{u}\right)$, we shrink $C_{u}$ until it just covers its farthest child and extend the circle of $p_{u}$ 's unique parent (height $\geq i$ ) by the same amount. This ensures that condition (2) is satisfied for the point $p_{u}$. Furthermore, this maintains all connections induced by $C_{u}$ before shrinking and does not increase the solution value $R$.

Using the observed optimal solution properties, we next describe an algorithm which will result in an optimal range assignment for $T$. 

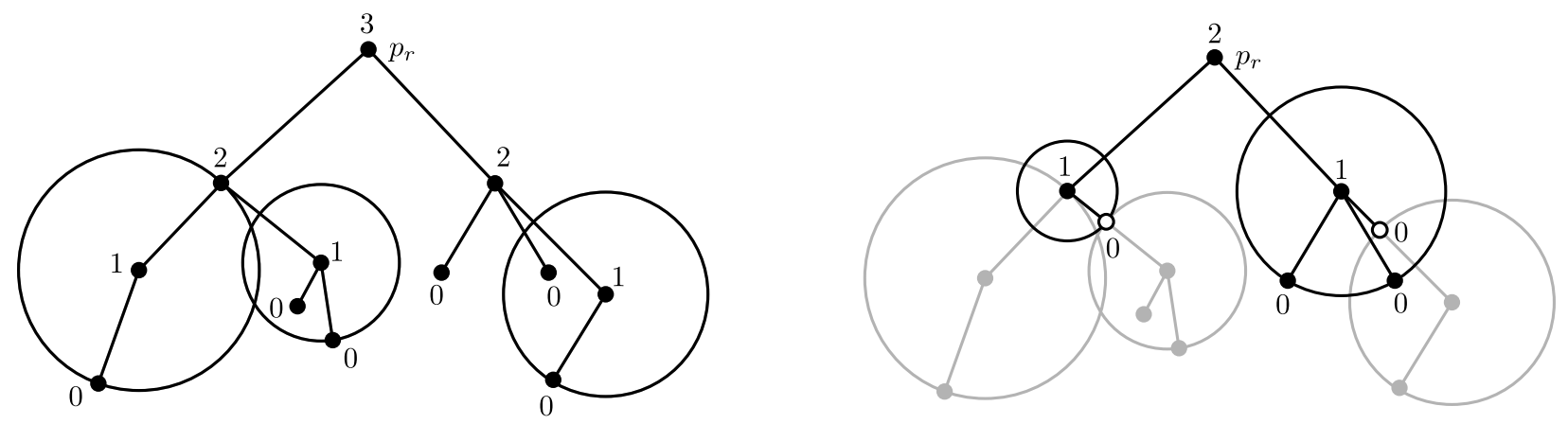

Figure 1: One step in algorithm 1 for a tree $T$ of height $h_{T}$ (left). The tree $\tilde{T}$ of height $h_{T}-1$ resulting from the remaining gaps is shown on the right.

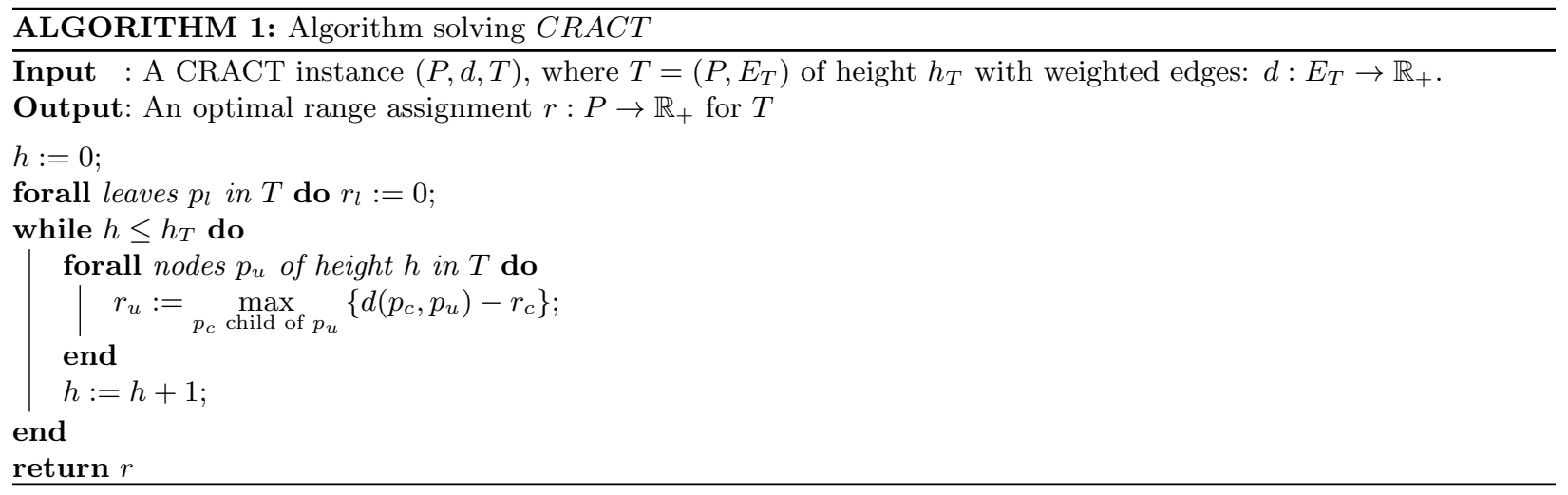

Theorem 3. CRACT can be solved in $O(n)$ time.

Proof. 1) [Correctness]. In the range assignment $r$ computed by Algorithm 1, a node $p_{u}$ at height $h$, $0<h \leq h_{T}$, in $T$ is assigned a radius $r_{u}$ where $r_{u}:=\max _{p_{c} \text { child of } p_{u}}\left\{d\left(p_{c}, p_{u}\right)-r_{c}\right\}$ and the leaves $p_{l}$ are assigned the radius $r_{l}=0$. The correctness of the algorithm now follows from Lemma 2

2) [Runtime]. As the height of any node in $T$ is unique for a fixed root, the algorithm assigns radii to each node in the tree exactly once. For every node $p$ of height $h \geq 1$, the computation of the required radius needs time proportional to its degree. During this computation, each edge in the tree (of which we have exactly $n-1$ ) is considered exactly once in total for the computation. Hence, the algorithm determines an optimal range assignment in $O(n)$ steps. Note that even if the height of the nodes cannot be gained directly from the data structure, it can be computed via one iteration over the tree, which again only requires linear time.

\section{NP-hardness for Bounded Radii}

In this section, we show that, if an upper bound of $\beta$ on the radii is also specified, the problem CRA becomes NP-hard using a reduction from 3-SAT. More formally, we are given a finite set $P$ of objects, the pairwise distances of the elements in $P$ measured by metric $d$ and an upper bound $\beta$ on the radii. The goal is to find an assignment of radii to $P$, such that the objective function $R=\sum_{i} r_{i}$ is minimized, subject to the constraint that $H$ is connected and $r_{i} \leq \beta$ for all $i$.

Definition 1 (3-SAT). In logic, a literal is a single (possibly negated) variable. A disjunction of three literals is called a clause $\gamma_{i}$. Given a Boolean formula $\mathcal{F}=\gamma_{1} \wedge \gamma_{2} \wedge \ldots \wedge \gamma_{c}$ that is a conjunction of $c$ 
clauses formed by a set of $v$ variables, the 3-Satisfiability problem (3-SAT) is to decide whether there exists an assignment of truth values to each of the $v$ variables, such that $\mathcal{F}$ evaluates to true. The variable-clause incidence graph consists of a vertex for each variable and each clause; an edge exists between a clause vertex representing a clause $c_{j}$ and a variable vertex representing a variable $x_{i}$, iff variable $x_{i}$ occurs in clause $c_{j}$. A 3-SAT instance is planar if the clause-variable incidence graph is planar.

It was shown by Lichtenstein [19] that 3-SAT is NP-complete, even when restricted to planar instances. This is the basis for many NP-completeness reductions for planar graphs and for point sets. In the following, we give a proof of NP-hardness of the problem CRA with bounded radii in the graph setting.

Theorem 4. With radii bounded by some constant $\beta$, the problem CRA is NP-hard for planar weighted graphs.

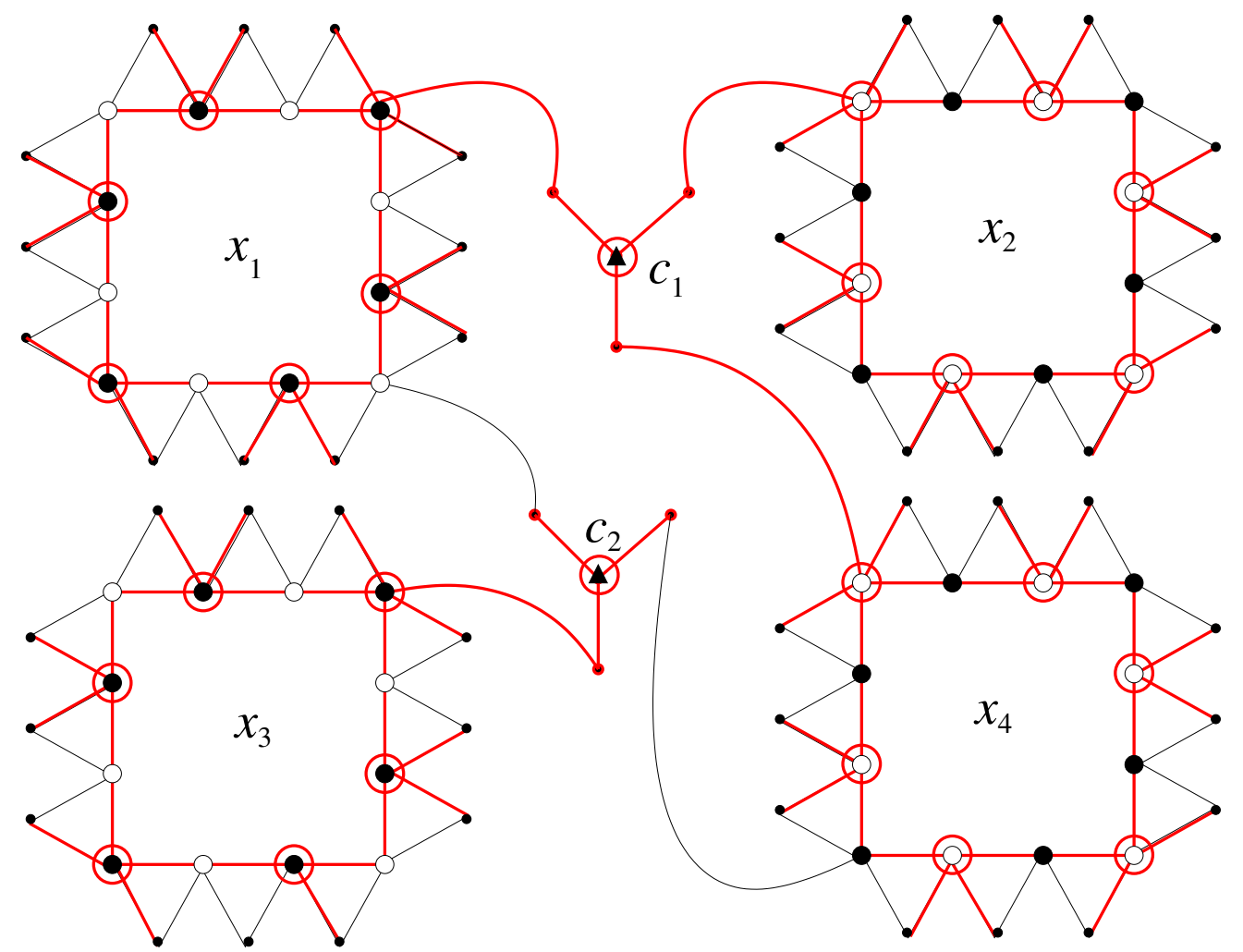

Figure 2: A graph representation for the 3SAT instance $\left(\bar{x}_{1} \vee x_{2} \vee \bar{x}_{3}\right) \wedge\left(x_{1} \vee \bar{x}_{2} \vee \bar{x}_{4}\right)$. All displayed edges have length $\beta$. "True" and "false" vertices are marked in bold white or black, respectively; auxiliary vertices are indicated by small dots. Clause vertices are indicated by small triangles, while the subgraphs for variables are labeled by variable names. Marked in red are the vertices assigned with a radius of $\beta$, as well as the corresponding connecting edges.

Proof. See Fig. 2 for the main ideas behind the construction. The proof uses a reduction from PlanaR 3SAT. Variables are represented by closed "loops" at distance $\beta$. Additional "connectivity" edges ensure that all variable gadgets are connected. Each clause is represented by a star-shaped set of four points which is connected to each corresponding variable loop over a $\beta$-edge. We claim that there is an optimal solution for the constructed CRA instance " $I_{C R A}\left(I_{3 S a t}\right)$ " with $\operatorname{cost} R=v \cdot 6 \beta+c \cdot \beta$ if and only if there is a satisfying variable assignment for the corresponding $3 \mathrm{SAT}$ instance $I_{3 S a t}$ with $v$ variables and $c$ clauses. 
" $\Rightarrow "$ : To verify that claim we first state some properties of solutions to an $I_{C R A}\left(I_{3 S a t}\right)$ CRA instance:

1. There are exactly two different feasible connected radii assignments of value $6 \beta$ for each variable loop. To make this clear see the triangle in Fig. 3 which illustrates that only "non-fractional" solutions provide the required sum of radii. More precisely, if more than one disk is used, the sum of radii either exceeds $\beta$ or one point remains uncovered, as the sum of each two radii must be greater or equal to $\beta$. For symmetric reasons this argument yields two valid "integral" $6 \beta$ solutions for each entire variable loop.
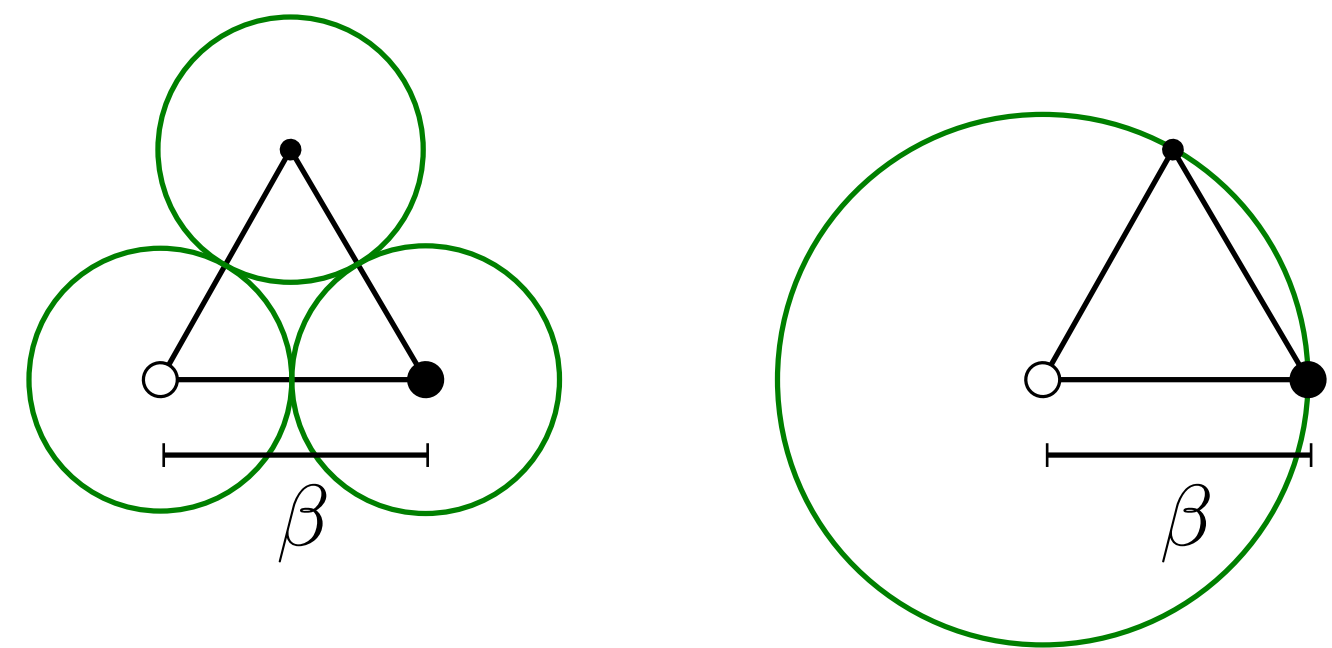

Figure 3: The triangular parts of the variable gadgets. A coverage by multiple disks (left) always costs more the a coverage with one disk (right).

2. The four nodes of a clause can be connected to the rest with cost at most $\beta$ only if there is at least one satisfying variable.

Since every clause star center $p_{c}$ can only be covered by disks around points that are not part of the variable loops, the only way to connect the star centers to the remainder with cost at most $\beta$ for each clause, is to use exactly one $\beta$-disk centered at one of the four corresponding clause nodes. Using $p_{c}$ as the $\beta$-disk center we must have a satisfying variable loop that closes the gap (of length $\beta$ ) between the clause and the remainder of the graph. Moreover, if we use a different node of the clause as the $\beta$-disk center, the remaining two uncovered star nodes must also be covered by disks from the variable loops; see Fig. 4 for illustration.

From the first property we know that the loop construction directly provides a lower bound of $v \cdot 6 \beta$ for the costs of the total variable loop coverage. Thus, only costs of $c \cdot \beta$ remain for the coverage of the clauses with total costs $R=v \cdot 6 \beta+c \cdot \beta$. Combined with the second property we conclude that there is a solution to $I_{C R A}\left(I_{3 S a t}\right)$ with cost $R=v \cdot 6 \beta+c \cdot \beta$ only if there is a satisfying variable assignment for $I_{3 S a t}$.

"Æ": Given a solution to $I_{3 S a t}$. We have seen that any satisfying variable assignment directly encodes a variable loop coverage in $I_{C R A}\left(I_{3 S a t}\right)$ with cost $v \cdot(6 \beta)$. If there is a variable that is not needed (i.e., one for which the value does not impact satisfiability of the clauses), it can be either treated as a normal "true" or "false" variable and is automatically connected to another variable loop via the "connectivity edges". The clause stars can each be covered with a $\beta$-disk centered at the clause star center $p_{c}$. Thereby, they are all connected to the remainder as we have at least one connecting (satisfying) variable loop for each clause. This construction $\operatorname{costs} c \cdot \beta$. In total, for every solution to $I_{3 S a t}$ we get a solution with the desired costs for $I_{C R A}\left(I_{3 S a t}\right)$.

The main reason why this proof cannot be extended to the case of unbounded radii is that we consider the objective function $Q_{1}=\sum r_{i}$ which does not prefer smaller disks as any $Q_{\alpha}$ with $\alpha>1$ does. That 

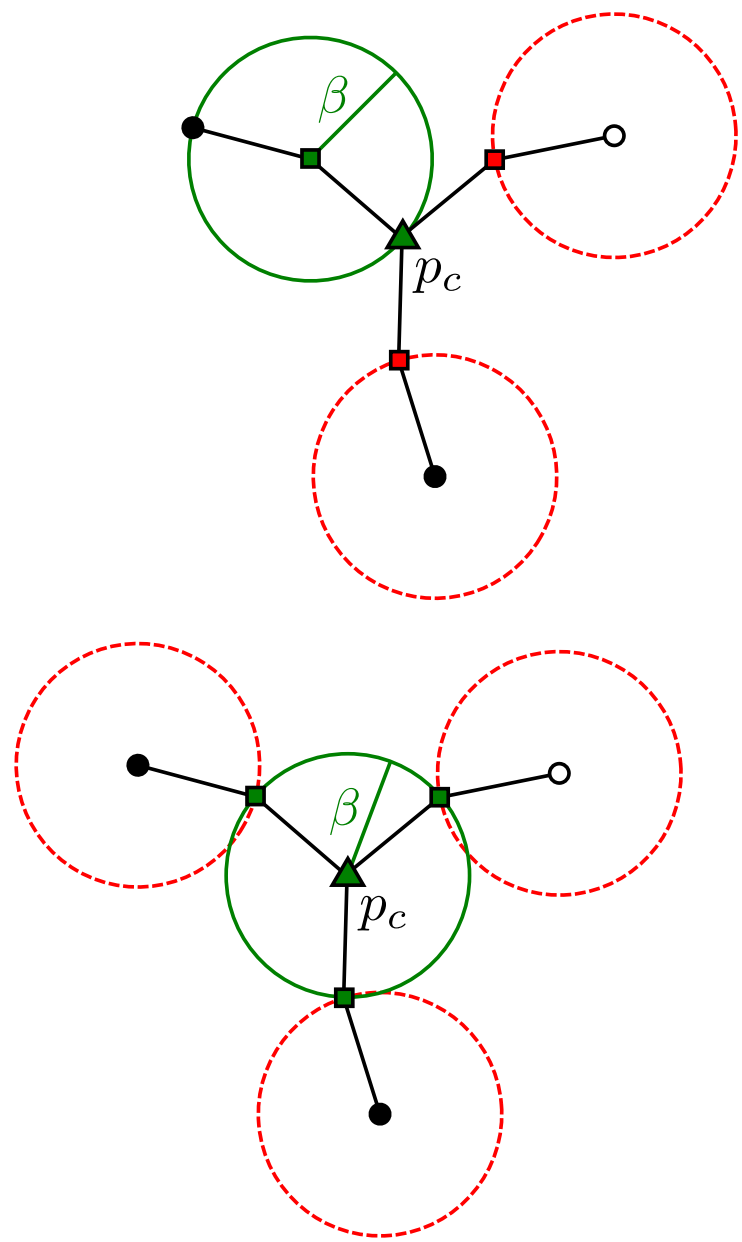

Figure 4: We cover each clause star by a $\beta$-disk centered at one of the inner nodes.

is, although we can construct some local loops that allow only two different integral optimal solutions, we are unable to see a way to connect these gadgets guaranteeing the absence of an optimal global 1-disk solution.

For the case of bounded radii, we can also show an NP-hardness proof for a geometric setting; again the proof uses a reduction to planar 3-Satisfiability. For this, we construct variable gadgets that each consist of disk-shaped point clusters. At the boundary of these clusters we uniformly arrange points such that exactly every third point is used as a disk center in an optimal solution. Chains of $\beta$-distant points ensure interior connectivity of the variable gadgets. (This is completely analogous to the construction for the proof of NP-completeness of packing squares into a polygonal domain presented as Theorem 1 in the paper by Baur and Fekete 4.) The clause gadgets are constructed as in the graph case. Additional point chains connect them to the corresponding variable loops.

\section{Solutions with a Limited Number of Circles}

A natural class of solutions arises when only a limited number of $k$ disks may have positive radius. In this section we show that these $k$-disk solutions already yield good approximations; we start by giving a class of lower bounds. 
We first observe that the number of disks needed for an optimal solution cannot be bounded by a constant number in general.

Theorem 5. For any integer $k \geq 1$, there is an instance of $n=k+3$ points in the plane that need exactly $k+1$ disks to be covered optimally.

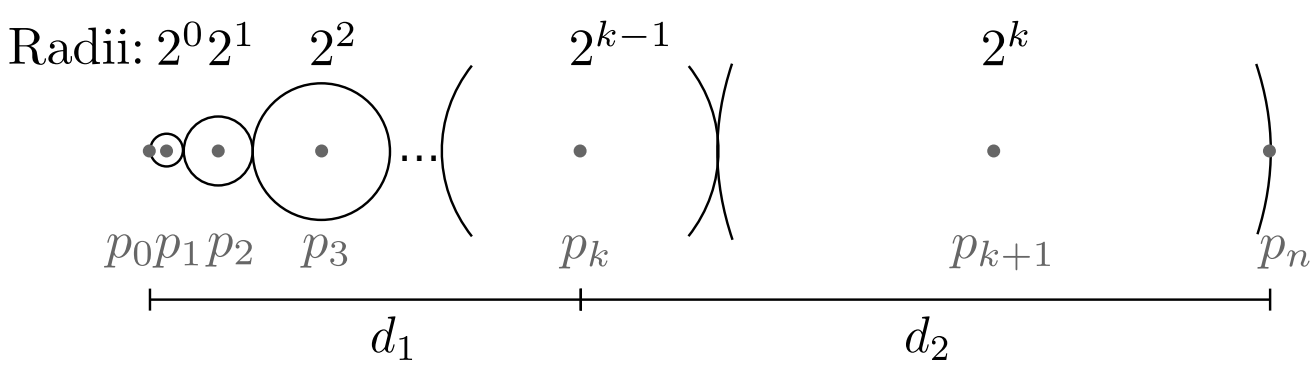

Figure 5: A class of CRA instances that need $k+1$ disks in an optimal solution.

Proof. Consider the example in Fig. 5 with $n=k+3$ points. The provided solution $R^{*}$ with $k+1$ disks is optimal, as $R^{*}:=\sum r_{i}^{*}=\frac{\operatorname{dist}\left(p_{0}, p_{n}\right)}{2}$. To show that the best $k$-disk solution is not optimal, we make the following observation. For any integer $k \geq 1$, we have $d_{1}=d\left(p_{0}, p_{k}\right)=2 \cdot \sum_{i=0}^{k-2} 2^{i}+2^{k-1}<2 \cdot 2^{k}+2^{k-1}=$ $d\left(p_{k}, p_{n}\right)=d_{2}$. Therefore, any $k$-disk solution that does not use point $p_{k+1}$ cannot be optimal, as the sum of the radii would be strictly greater than $R^{*}$. On the other hand, if the $k$-disk solution contains a disk centered at $p_{k+1}$, it must be of radius $r_{k+1}^{*}$ in order to be optimal. This results in a set of $n-1$ points to be connected optimally using $k-1$ disks. Hence, using induction on $k$, we conclude that exactly $k+1$ disks are needed.

In the following we establish upper and lower bounds on the approximation ratios of 1- and 2-disk solutions for CRA.

Lemma 6. Let $\mathcal{P}$ be a longest (simple) path in a connectivity graph corresponding to an optimal solution $R^{*}$, and let $e_{m}$ be an edge in $\mathcal{P}$ containing the midpoint of $\mathcal{P}$. Then $R^{*}=\sum r_{i}^{*} \geq \max \left\{\frac{1}{2}|\mathcal{P}|,\left|e_{m}\right|\right\}$.

This follows directly from the following simple property of the connectivity graph: In this graph, for any edge $e=p_{u} p_{v}$ with length $|e|$ in $\mathcal{P}, r_{u}+r_{v} \geq|e|=d\left(p_{u}, p_{v}\right)$.

Theorem 7. A best 1-disk solution for CRA is a $\frac{3}{2}$-approximation, even in the graph version of the problem.

Proof. Consider a longest path $\mathcal{P}=\left(p_{0}, \ldots, p_{k}\right)$ of length $|\mathcal{P}|=d_{\mathcal{P}}\left(p_{0}, \ldots, p_{k}\right):=\sum_{i=0}^{k-1}\left|p_{i} p_{i+1}\right|$ in the connectivity graph of an optimal solution. Let $R^{*}:=\sum r_{i}^{*}$ be the cost of the optimal solution, and $e_{m}=p_{i} p_{i+1}$ as in Lemma 6 . Let $\bar{d}_{i}:=d_{\mathcal{P}}\left(p_{i}, \ldots, p_{k}\right)$ and $\bar{d}_{i+1}:=d_{\mathcal{P}}\left(p_{0}, \ldots, p_{i+1}\right)$. Then $d_{\min }:=$ $\min \left\{\bar{d}_{i}, \bar{d}_{i+1}\right\} \leq \frac{\bar{d}_{i}+\bar{d}_{i+1}}{2}=\frac{d_{\mathcal{P}}\left(p_{0}, \ldots, p_{i}\right)+2\left|e_{m}\right|+d_{\mathcal{P}}\left(p_{i+1}, \ldots, p_{k}\right)}{2}=\frac{|\mathcal{P}|}{2}+\frac{\left|e_{m}\right|}{2} \leq R^{*}+\frac{R^{*}}{2}=\frac{3}{2} R^{*}$. So a single disk $C$ with a radius of $d_{\min } \leq \frac{3}{2} R^{*}$ around the point in $P$ that is nearest to the middle of path $\mathcal{P}$ covers $\mathcal{P}$. Without loss of generality, let this center point of $C$ be $p_{i+1}$, then $d_{\min }=\bar{d}_{i+1}$. We claim that the chosen disk $C$ also covers all points in $P$. This can be seen by assuming the existence of a point $p^{\prime} \in P$ that is not covered by $C$; see Fig. 6. Because the optimal solution must induce a connected connectivity graph, there is a path $\mathcal{P}^{\prime}$ from $p^{\prime}$ to a node $p_{j}$ in $\mathcal{P}$ that has no edge in common with $\mathcal{P}$, i.e. $V\left(\mathcal{P}^{\prime}\right) \cap V(\mathcal{P})=\left\{p_{j}\right\}$. Without loss of generality, let $p_{j}$ lie between $p_{0}$ and $p_{i}$ in $\mathcal{P}$. If $p^{\prime}$ is not covered by $C$, the path $\mathcal{P}^{\prime}$ must be longer than the path segment $\left(p_{0}, \ldots, p_{j}\right)$ of $\mathcal{P}$. We get a path $\mathcal{P}^{\prime} \cup\left(p_{j}, \ldots, p_{k}\right)$ with length greater than $|\mathcal{P}|$, which contradicts the maximality of $\mathcal{P}$.

Fig. 7 shows that this bound is tight. There is a 2-disk solution of cost 2 while the best 1-disk solution has cost 3 . We now show that using two disks yields an even better approximation factor. 


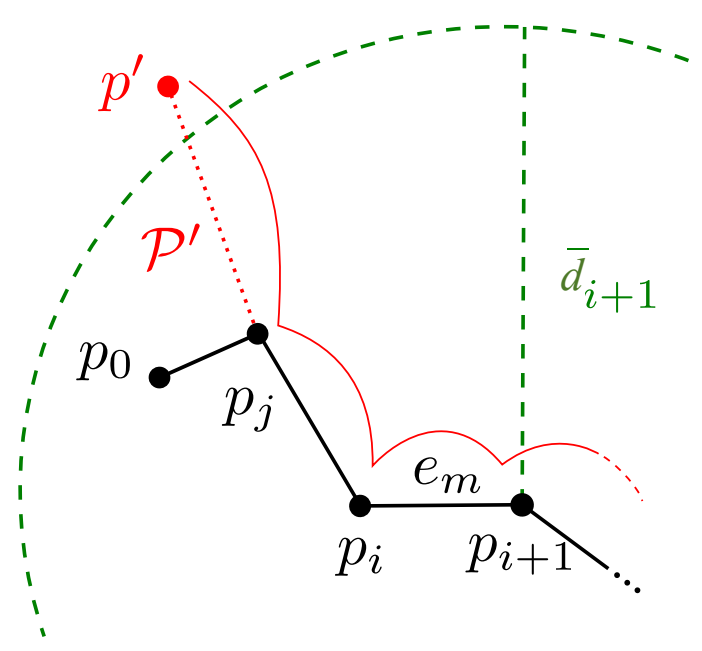

Figure 6: The presence of an uncovered point $p^{\prime}$ (red) implies the existence of a longer path $\mathcal{P}^{\prime} \cup\left(p_{j}, \ldots, p_{k}\right)$ (sketched by the curved red line).

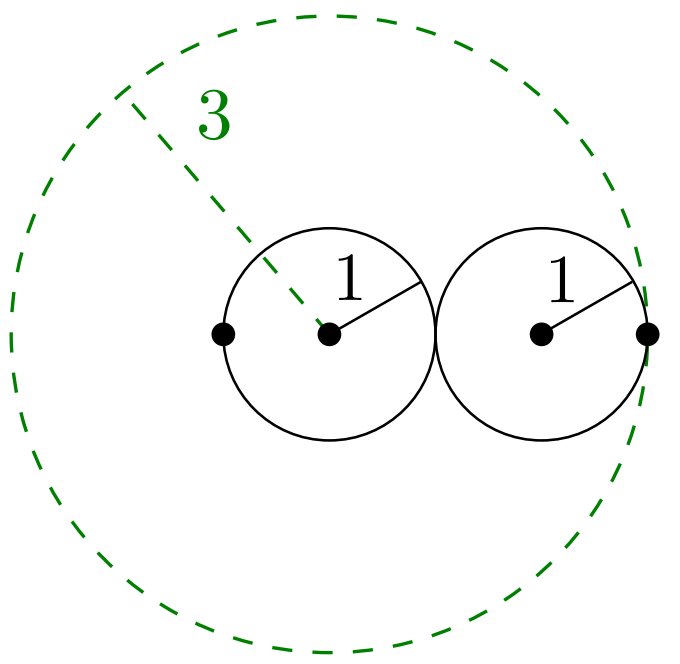

Figure 7: A lower bound of $\frac{3}{2}$ for 1-disk solutions.

Theorem 8. A best 2-disk solution for CRA is a $\frac{4}{3}$-approximation, even in the graph version of the problem.

Proof. Let $\mathcal{P}=\left(p_{0}, \ldots, p_{k}\right)$ be a longest path of length $|\mathcal{P}|=d_{\mathcal{P}}\left(p_{0}, \ldots, p_{k}\right):=\sum_{i=0}^{k-1}\left|p_{i} p_{i+1}\right|$ in the connectivity graph of an optimal solution with radii $r_{i}^{*}$. Then by Lemma 6, $R^{*}:=\sum r_{i}^{*} \geq \frac{1}{2}|\mathcal{P}|$. We distinguish two cases; see Fig. 8 .

Case 1. There is a point $x$ on $\mathcal{P}$ at a distance of at least $\frac{1}{3}|\mathcal{P}|$ from both endpoints. Then there is a 1-disk solution that is a $\frac{4}{3}$-approximation.

Case 2. There is no such point $x$. Then two disks are needed. One of them is placed at a point in the first third of $\mathcal{P}$, and the other disk is placed at a point in the last third of $\mathcal{P}$. Let $e_{m}=p_{i} p_{i+1}$ be defined as in Lemma 6. Further, let $d_{i}:=d_{\mathcal{P}}\left(p_{0}, \ldots, p_{i}\right)$, and let $d_{i+1}:=d_{\mathcal{P}}\left(p_{i+1}, \ldots, p_{k}\right)$. Then $\left|e_{m}\right|=|\mathcal{P}|-d_{i}-d_{i+1}$ and $d_{i}, d_{i+1}<\frac{1}{3}|\mathcal{P}|$. 

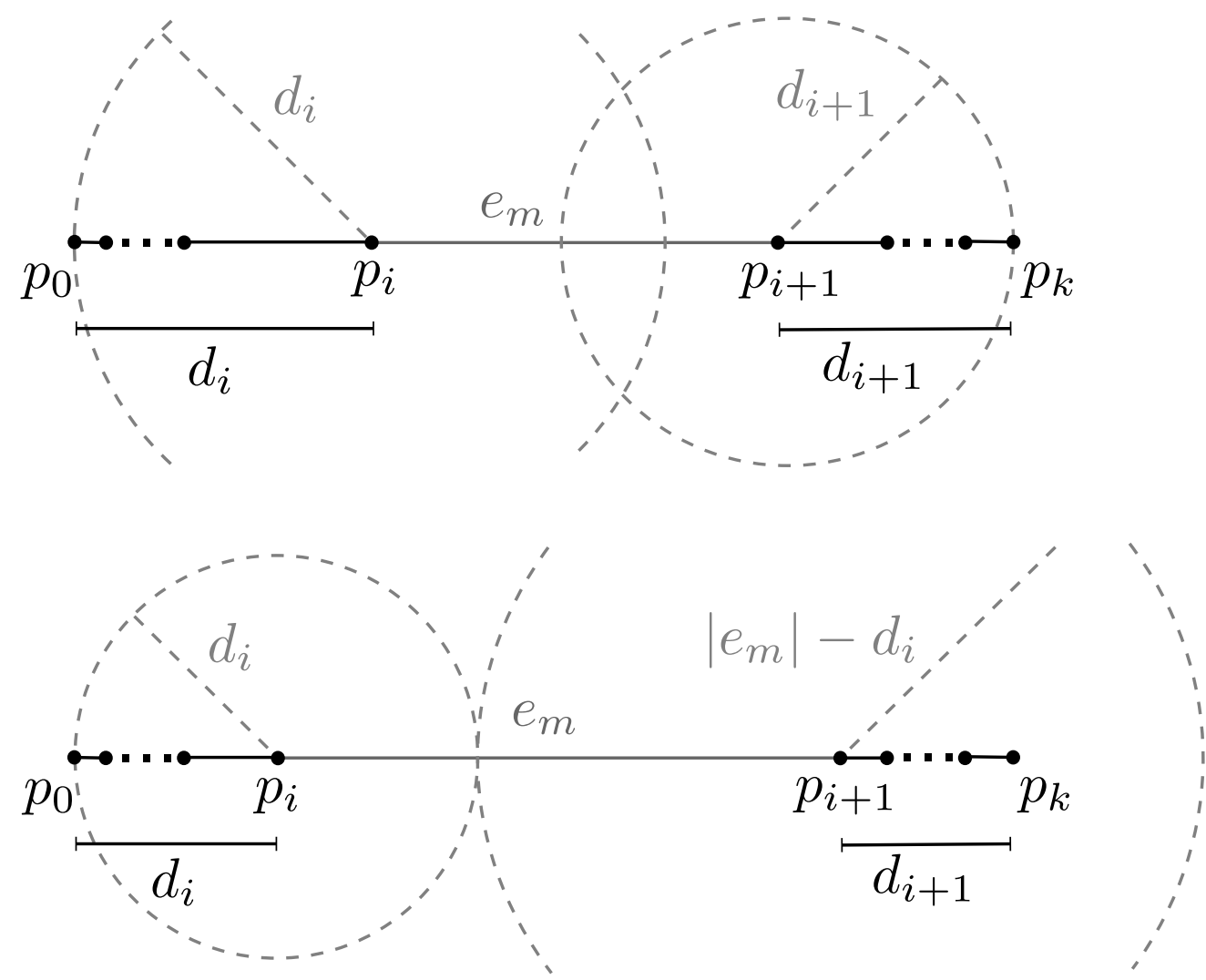

Figure 8: Two $\frac{4}{3}$-approximate 2-disk solutions (light grey, dashed): (Top) Case 2a, when $\left|e_{m}\right|<d_{i}+d_{i+1}$; (bottom) Case 2b, where $\left|e_{m}\right|>d_{i}+d_{i+1}$.

Case 2a. If $\left|e_{m}\right|<\frac{1}{2}|\mathcal{P}|$ then $d_{i}+d_{i+1}=|\mathcal{P}|-\left|e_{m}\right|>\frac{1}{2}|\mathcal{P}|>\left|e_{m}\right|$. Set $r_{i}:=d_{i}$ and $r_{i+1}:=d_{i+1}$, then the path is covered. Analogously to the proof of Theorem 7, we conclude that there is no point $p^{\prime} \in P$ uncovered by the chosen disks, as there will otherwise be a longer path than $\mathcal{P}$. Since $d_{i}, d_{i+1}<\frac{1}{3}|\mathcal{P}|$ we have $r_{i}+r_{i+1}=d_{i}+d_{i+1}<\frac{2}{3}|\mathcal{P}| \leq \frac{4}{3} R^{*}$ and the claim holds.

Case 2b. Otherwise, if $\left|e_{m}\right| \geq \frac{1}{2}|\mathcal{P}|$ then $d_{i}+d_{i+1} \leq \frac{1}{2}|\mathcal{P}| \leq\left|e_{m}\right|$. Assume $d_{i} \geq d_{i+1}$. Choose $r_{i}:=d_{i}$ and $r_{i+1}:=\left|e_{m}\right|-d_{i}$. As $d_{i+1} \leq\left|e_{m}\right|-d_{i}$ the path $\mathcal{P}$ is covered. Again the existence of an uncovered point contradicts the maximality of $\mathcal{P}$. Finally, $r_{i}+r_{i+1}=d_{i}+\left(\left|e_{m}\right|-d_{i}\right)=\left|e_{m}\right|$, which is the lower bound and thus the range assignment is optimal.

If all points of $P$ lie on a straight line, the approximation ratio for two disks can be improved. We first observe an interesting property of optimal solutions in this case.

Lemma 9. Let $P$ be a set of points on a straight line. Then there is a non-overlapping optimal solution, i.e., one in which all disks have disjoint interior.

Proof. An arbitrary optimal solution is modified as follows. For every two overlapping disks $C_{i}$ and $C_{i+1}$ with centers $p_{i}$ and $p_{i+1}$, we decrease $r_{i+1}$, such that $r_{i}+r_{i+1}=\operatorname{dist}\left(p_{i}, p_{i+1}\right)$, and increase the radius of $C_{i+2}$ by the same amount. This can be iterated, until there is at most one overlap at the outermost disk $C_{j}$ (with $C_{j-1}$ ). Then there must be a point $p_{j+1}$ on the boundary of $C_{j}$ : otherwise we could shrink $C_{j}$ contradicting optimality. Decreasing $C_{j}$ 's radius $r_{j}$ by the overlap $l$ and adding a new disk with radius $l$ around $p_{j+1}$ creates an optimal solution without overlap. 
Theorem 10. Let $P$ be a set of points on a straight line $g$. Then, a best 2-disk solution for CRA is a $\frac{5}{4}$-approximation.

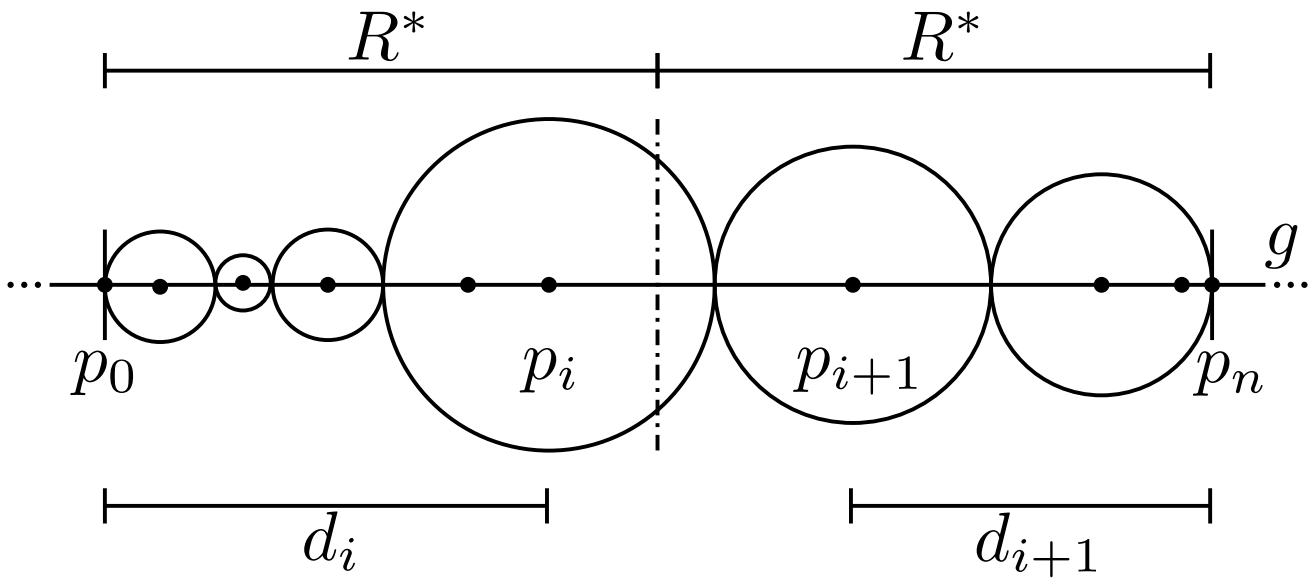

Figure 9: A non-overlapping optimal solution.

Proof. According to Lemma 9, we are, without loss of generality, given an optimal solution $R^{*}$ with nonoverlapping disks. Let $p_{0}$ and $p_{n}$ be the outermost intersection points of the optimal solution disks and $g$. Without loss of generality, we may further assume $p_{0}, p_{n} \in P$ and $R^{*}:=\sum r_{i}^{*}=\frac{\operatorname{dist}\left(p_{0}, p_{n}\right)}{2}$ (otherwise, we can add the outermost intersection point of the outermost disk and $g$ to $P$, which may only improve the approximation ratio). Let $p_{i}$ denote the rightmost point in $P$ left to the middle of $\overline{p_{0} p_{n}}$ and let $p_{i+1}$ its neighbor on the other half. Further, let $d_{i}:=\operatorname{dist}\left(p_{0}, p_{i}\right), d_{i+1}:=\operatorname{dist}\left(p_{i+1}, p_{n}\right)$ (See Fig. 9). Assume, $d_{i} \geq d_{i+1}$. We now give $\frac{5}{4}$-approximate solutions using one or two disks that cover $\overline{p_{0} p_{n}}$.

Case 1. If $\frac{3}{4} R^{*} \leq d_{i}$ then $\frac{5}{4} R^{*} \geq 2 R^{*}-d_{i}=\operatorname{dist}\left(p_{i}, p_{n}\right)$. Thus, the solution consisting of exactly one disk with radius $2 R^{*}-d_{i}$ centered at $p_{i}$ is sufficient.

Case 2. If $\frac{3}{4} R^{*}>d_{i} \geq d_{i+1}$ we need two disks to cover $\overline{p_{0} p_{n}}$ with $\frac{5}{4} R^{*}$.

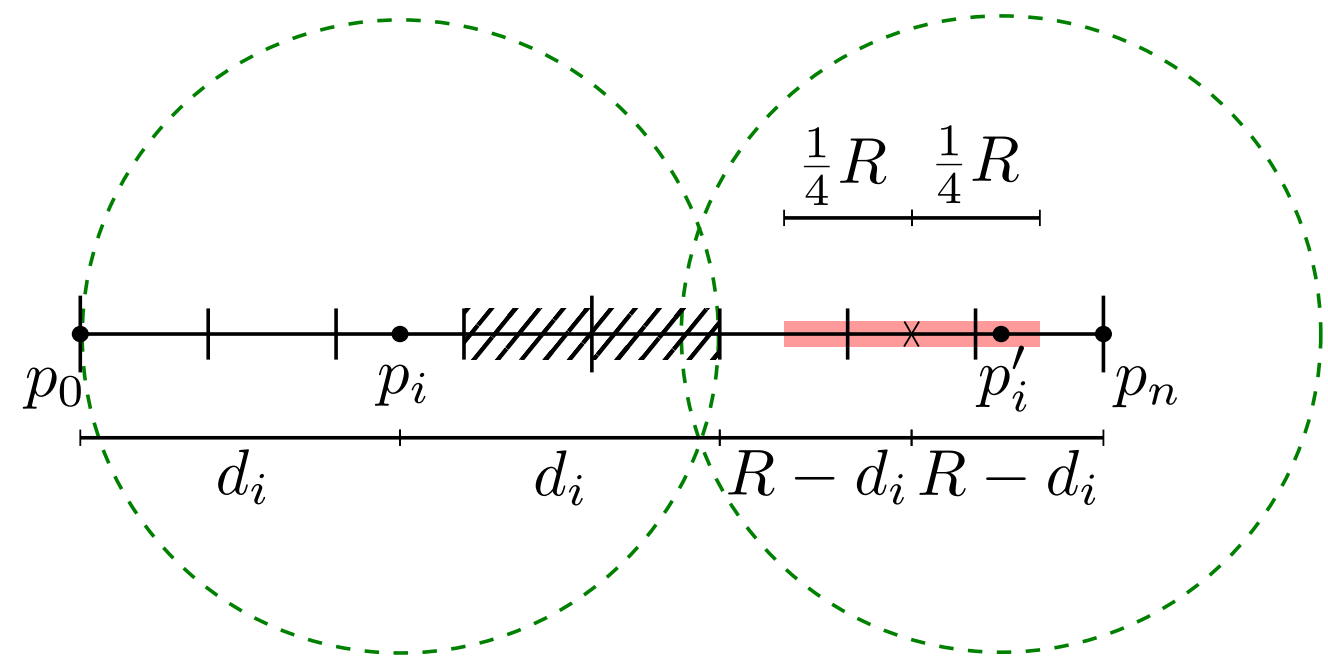

Figure 10: A $\frac{5}{4}$-approximate 2-disk solution with $d_{i}<\frac{3}{4} R^{*}$. The cross marks the position of the optimal counterpart $p_{i}^{*}$ to $p_{i}$ and the grey area sketches $A_{i}$.

Case 2a. The point $p_{i}$ could be a center point of an optimal two-disk solution if there was a point $p_{i}^{*}$ with $\operatorname{dist}\left(C_{i}, p_{i}^{*}\right)=\operatorname{dist}\left(p_{i}^{*}, p_{n}\right)=R^{*}-d_{i}$. So in case there is a $p_{i}^{\prime} \in P$ that lies in a $\frac{1}{4} R^{*}$-neighborhood of 
such an optimal $p_{i}^{*}$ we get $\operatorname{dist}\left(C_{i}, p_{i}^{\prime}\right), \operatorname{dist}\left(p_{i}^{\prime}, p_{n}\right) \leq R^{*}-d_{i}+\frac{1}{4} R^{*}$ (see Fig. 10). Thus, $r\left(p_{i}\right):=d_{i}, r\left(p_{i}^{\prime}\right):=$ $R^{*}-d_{i}+\frac{1}{4} R^{*}$ provides a $\frac{5}{4}$-approximate solution.

Case 2b. Analogously to Case $2 \mathrm{a}$, there is a point $p_{i+1}^{\prime} \in P$ within a $\frac{1}{4} R^{*}$-range of an optimal counterpart to $p_{i+1}$. Then we can take $r\left(p_{i+1}\right):=d_{i+1}, r\left(p_{i+1}^{\prime}\right):=R^{*}-d_{i+1}+\frac{1}{4} R^{*}$ as a $\frac{5}{4}$-approximate solution.

Case 2c. Assume that there is neither such a $p_{i}^{\prime}$ nor such a $p_{i+1}^{\prime}$. Since $d_{i}, d_{i+1}$ are in $\left(\frac{1}{4} R^{*}, \frac{3}{4} R^{*}\right)$, we have $\frac{1}{4} R^{*}<R^{*}-d_{j}<\frac{3}{4} R^{*}$ for $j=i, i+1$, which implies that there are two disjoint areas $A_{i}, A_{i+1}$, each with diameter equal to $\frac{1}{2} R^{*}$ and excluding all points of $P$. Since $p_{i}$, the rightmost point on the left half of $\overline{p_{0} p_{n}}$, has a greater distance to $A_{i}$ than to $p_{0}$, any disk around a point on the left could only cover parts of both $A_{i}$ and $A_{i+1}$ if it has a greater radius than its distance to $p_{0}$. This contradicts the assumption that $p_{0}$ is a leftmost point of a disk in an optimal solution. The same applies to the right-hand side. Thus, $A_{i} \cup A_{i+1}$ must contain at least one point of $P$, and therefore one of the previous cases leads to a $\frac{5}{4}$-approximation.

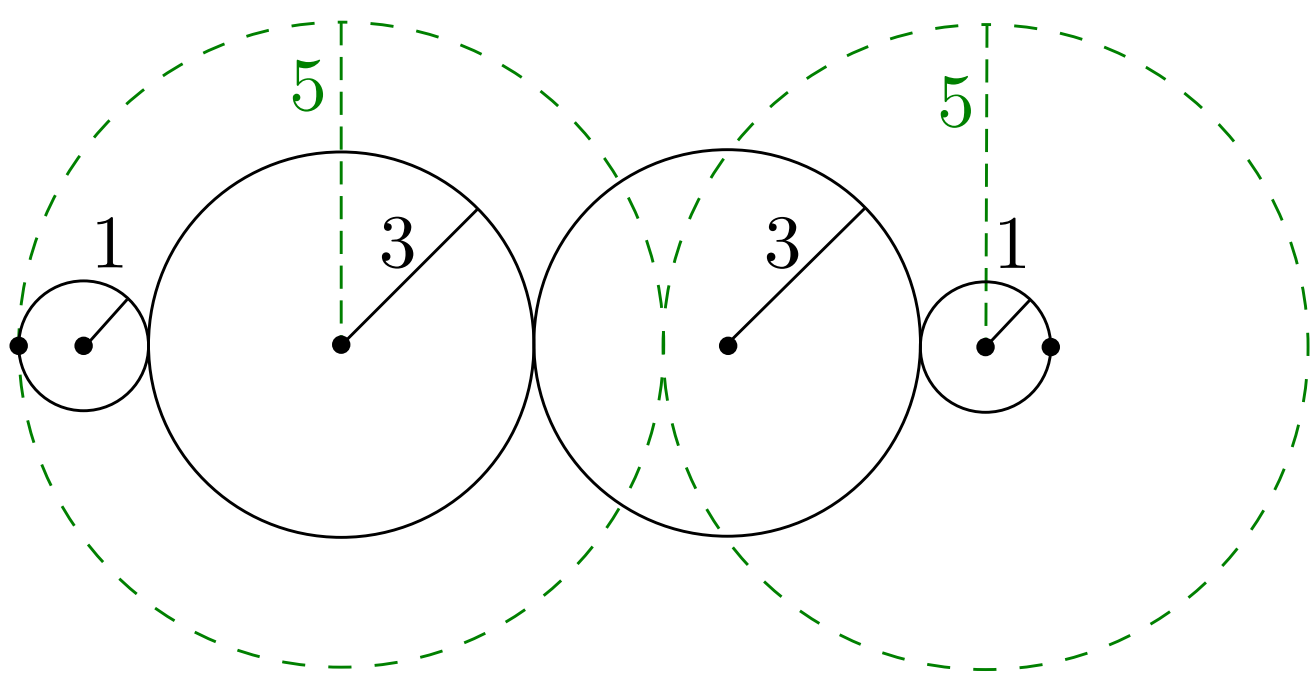

Figure 11: A lower bound of $\frac{5}{4}$ for 2-disk solutions.

Fig. 11 shows that the bound is tight. We believe that this is also the worst case when points are not on a line. Indeed, the solutions constructed in the proof of Theorem 10 cover a longest path $\mathcal{P}$ in an optimal solution for a general $P$. If this longest path consists of at most three edges, $p_{i}\left(=: p_{i+1}^{\prime}\right)$ and $p_{i+1}\left(=: p_{i}^{\prime}\right)$ can be chosen as disk centers, covering all of $P$. However, if $\mathcal{P}$ consists of at least four edges, a solution for the diameter may produce two internal non-adjacent center points that do not necessarily cover all of $P$.

\section{A Polynomial-Time Approximation Scheme for CRA}

We have seen (Theorem 4. Section 3) that the problem CRA is NP-hard when there are upper bounds given on the possible radii, $r_{i}$, of the input points $p_{i} \in P$, even if these upper bounds are all the same value $\beta$. We do not yet know the complexity of exactly solving the problem CRA in the case in which the radii $r_{i}$ are not bounded above. In this section, we give a polynomial-time approximation scheme (PTAS) for CRA for this case $(\beta=\infty)$ : for any fixed $\epsilon>0$, we compute, in polynomial time, a set of radii, $r_{i}$, so that the disks of radii $r_{i}$ centered at the respective input points $p_{i}$ form a connected set of disks, and the sum of radii $\sum_{i} r_{i}$ is at most $(1+\epsilon)$ times optimal.

To design a PTAS for this problem, we first prove a structure theorem that allows us to apply the $m$ guillotine method ([22]) and transform any solution into an " $m$-guillotine solution" (defined precisely below) with a small increase in the sum of radii. This theorem permits the subsequent use of dynamic programming to obtain a PTAS. 


\subsection{Structural Results}

For our first structural result, we need some definitions. Consider a set $\mathcal{D}$ of disks, centered at the points $P=\left\{p_{1}, p_{2}, \ldots, p_{n}\right\}$. Let $\delta=\operatorname{diam}(P)$ denote the diameter of the point set; i.e., $\delta$ is the maximum of the (Euclidean) distances between pairs of points of $P$. If the radii of the disks $\mathcal{D}$ are all from the discrete set $\mathcal{R}=\{\delta / m n, 2 \delta / m n, \ldots, \delta\}$, then we will say that $\mathcal{D}$ is a set of $\mathcal{R}_{m, P}$-disks, or $\mathcal{R}$-disks, for short, with the understanding that $m=O(1 / \epsilon)$ and $P$ will be fixed throughout our discussion. Now consider a set $\mathcal{D}$ of $\mathcal{R}$-disks, centered on the points $P$. We let $\mathcal{I}_{x}$ (resp., $\mathcal{I}_{y}$ ) denote the set of $x$-coordinates (resp., $y$-coordinates) of the set of all coordinate-extreme points (leftmost, rightmost, topmost, and bottommost) of the disks $\mathcal{D}$. Specifically, $\mathcal{I}_{x}=\left\{x\left(p_{i}\right) \pm j(\delta / m n): 1 \leq i \leq n, 0 \leq j \leq m n\right\}$ and $\mathcal{I}_{y}=\left\{y\left(p_{i}\right) \pm j(\delta / m n): 1 \leq i \leq n, 0 \leq\right.$ $j \leq m n\}$, where $x(\cdot)$ and $y(\cdot)$ denote $x$ - and $y$-coordinates.

We begin with a simple lemma that shows that we can round up the radii of a feasible solution, to make it a set of $\mathcal{R}$-disks, at a small cost to the objective function:

Lemma 11. Let $\sum_{i} r_{i}$ be the sum of radii in a feasible (connected union) solution, $\mathcal{D}$. Then, for any fixed $\epsilon>0$, there exists a set, $\mathcal{D}_{m}$, of $n$ disks of radii $r_{i}^{\prime}$ centered on points $p_{i}$, such that $(a) . r_{i}^{\prime} \in \mathcal{R}=$ $\{\delta / m n, 2 \delta / m n, \ldots, \delta\}$, where $\delta=\operatorname{diam}(P)$ is the diameter of the input point set $P$ and $m=\lceil 2 / \epsilon\rceil$; and (b). $\sum_{i} r_{i}^{\prime} \leq(1+\epsilon) \sum_{i} r_{i}$

Proof. Each of the $n$ radii $r_{i}$ can be increased by at most $\delta / m n \leq \epsilon \delta / 2 n$ at a total cost (increase in the sum of all radii) of at most $\epsilon \delta / 2$. Since increasing the radii of the disks keeps the set of disks connected, and since $\sum_{i} r_{i} \geq \delta / 2$, we obtain the result.

Next, we state and prove a simple observation about the structure of an optimal solution:

Lemma 12. Let $\mathcal{D}$ be a set of circular disks of radii $r_{i}$ centered at points $p_{i} \in P$, such that the union of the disks is connected. Then, there exists a set $\mathcal{D}^{\prime}$ of circular disks centered at points $p_{i} \in P$, such that the union of the disks $\mathcal{D}^{\prime}$ is connected, the sum of the radii of disks $\mathcal{D}^{\prime}$ is at most $\sum_{i} r_{i}$, and no point in the plane lies within more than 6 disks of $\mathcal{D}^{\prime}$. In particular, in an optimal solution, no point in the plane lies within more than 6 disks of the set of optimal disks. Further, if $\mathcal{D}$ are $\mathcal{R}$-disks, then there exists a set $\mathcal{D}^{\prime}$ of $\mathcal{R}$-disks of total radii $\sum_{i} r_{i}$ having the property that no point in the plane lies within more than 6 disks of $\mathcal{D}^{\prime}$.

Proof. If some point $p$ lies within more than 6 disks of $\mathcal{D}$, then we know that two of them, say $C_{1}$ and $C_{2}$, have centers, $p_{1}$ and $p_{2}$, within a 60-degree cone with apex $p$. Thus, the distance $\left|p_{1} p_{2}\right|$ between the two centers is less than the larger, say $r_{1}$ (the radius of $C_{1}$ ), of the two radii $\left(r_{1}\right.$ and $\left.r_{2} \leq r_{1}\right)$, implying that center $p_{2}$ lies within the disk $C_{1}$. Thus, if we enlarge the disk $C_{1}$ to have radius $r_{2}+\left|p_{1} p_{2}\right| \leq r_{1}+r_{2}$, the enlarged disk completely covers disk $C_{2}$, allowing $C_{2}$ to be deleted (i.e., allowing the radius of $C_{2}$ to be shrunk to 0). This modification yields a set $\mathcal{D}^{\prime}$ of disks that are connected (since the union of the disks of $\mathcal{D}^{\prime}$ contains the union of the disks of $\mathcal{D}$ ), whose total sum of radii is at most that of the set $\mathcal{D}$.

If the disks $\mathcal{D}$ are $\mathcal{R}$-disks, then the same argument applies, but we now replace the radius $r_{1}$ with the enlarged radius $r_{1}+r_{2}\left(\geq r_{2}+\left|p_{1} p_{2}\right|\right)$, while shrinking $r_{2}$ to zero. Since the disks $\mathcal{D}$ are $\mathcal{R}$-disks, we know that $r_{1}, r_{2} \in \mathcal{R}=\{\delta / m n, 2 \delta / m n, \ldots, \delta\}$, and therefore $r_{1}+r_{2} \in \mathcal{R}$. Thus, disks $\mathcal{D}^{\prime}$ are $\mathcal{R}$-disks.

Our main structural result requires further definitions. Let $\mathcal{D}$ be a set of $n$ disks; we consider the disks to be closed (include their bounding circles). Let $\rho$ be an axis-parallel rectangle. An axis-parallel line $\ell$ that intersects $\rho$ is said to be a cut of $\rho$. A cut $\ell$ is said to be m-perfect with respect to $\mathcal{D}$ and the rectangle $\rho$ if $\ell$ intersects the interiors of at most $c_{1} m+c_{2}$ disks of $\mathcal{D}$ that lie interior to $\rho$, for fixed constants (independent of $m, \epsilon) c_{1}$ and $c_{2}$.

We say that a set $\mathcal{D}$ of $\mathcal{R}$-disks is $m$-guillotine with respect to (axis-aligned) rectangle $\rho$ if either (1) no disk of $\mathcal{D}$ lies interior to $\rho$, or (2) there exists an (axis-parallel) cut $\ell$, defined by coordinates $\mathcal{I}_{x}$ and $\mathcal{I}_{y}$, that is $m$-perfect with respect to $\mathcal{D}$ and $\rho$, such that $\mathcal{D}$ is $m$-guillotine with respect to both of the rectangles into which $\rho$ is partitioned by the cut $\ell$. We say that a set $\mathcal{D}$ of $\mathcal{R}$-disks is $m$-guillotine if $\mathcal{D}$ is $m$-guillotine with respect to the bounding box, $B B(\mathcal{D})$, of $\mathcal{D}$. See Fig. 12 for an example of a set of disks that is 1-guillotine. 


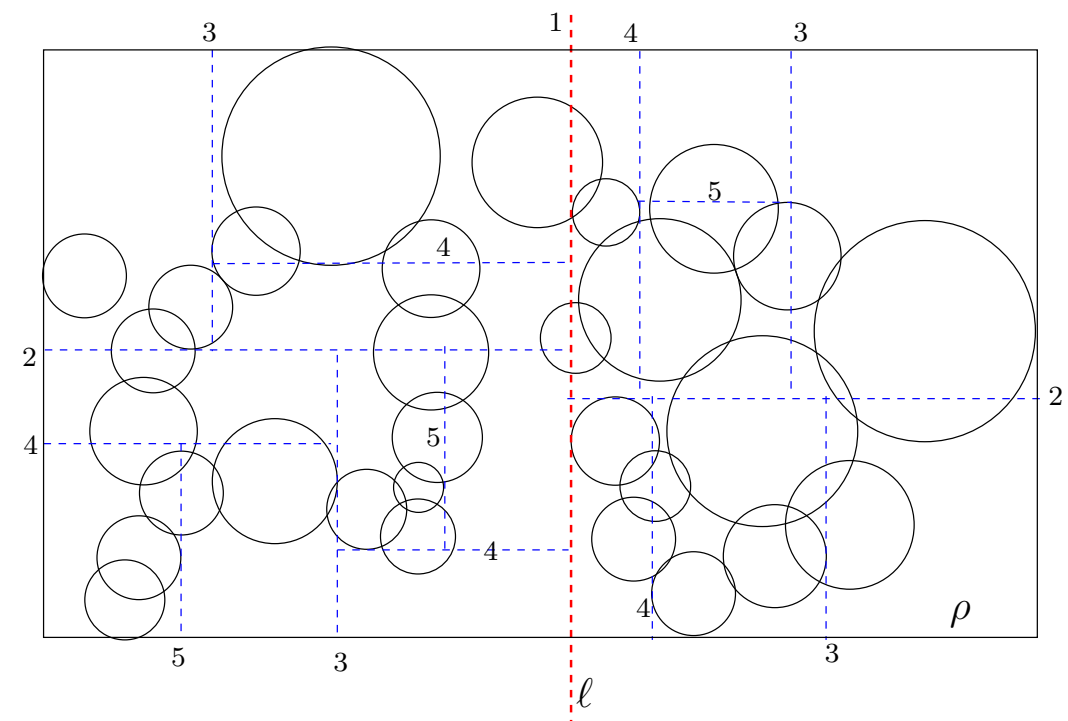

Figure 12: An example of an $m$-guillotine set of disks. The vertical red cut $\ell$ is the first cut (labelled "1") in the recursive decomposition of the rectangle $\rho$. The blue cuts occur in the recursive decomposition; each is labeled with an integer ("2" through "5") indicating the level in the recursive decomposition. Each cut intersects the interior of at most 2 disks that are interior to the rectangle corresponding to the cut. All cuts are at coordinates $\mathcal{I}_{x}$ or $\mathcal{I}_{y}$. In the final decomposition of $\rho$, no disk lies interior to a rectangular face (some do lie within a face, in contact with the boundary). Thus, the set of disks is 1-guillotine, with constants $c_{1}=2, c_{2}=0$.

We are now ready to state and prove our structure theorem, which shows that we can transform an arbitrary set $\mathcal{D}$ of circular disks centered on points $P$, having a connected union and a sum of radii $R=\sum_{i} r_{i}$, into an $m$-guillotine set of $\mathcal{R}$-disks, $\mathcal{D}_{m}$, having sum of radii at most $(1+\epsilon) R$ and having a connected union. More specifically, we show:

Theorem 13. Let $\mathcal{D}$ be a set of $n$ circular disks of radii $r_{i}$ centered at points $p_{i} \in P$, such that the union of the disks is connected. Then, for any fixed $\epsilon>0$, there exists an m-guillotine set $\mathcal{D}_{m}$ of at most $n \mathcal{R}$-disks such that the union of the circular disks $\mathcal{D}_{m}$ is connected and the sum of the radii of disks of $\mathcal{D}_{m}$ is at most $(1+(C / m)) \sum_{i} r_{i}$. Here, $m=\lceil 2 / \epsilon\rceil$ and $C$ is a constant.

Proof. First, by Lemma 12, we can assume that no point in the plane lies in more than 6 of the input disks.

Consider the set of bounding boxes (squares) of the disks $\mathcal{D}$. For each disk of radius $r_{i}$, we consider the 4 edges of its bounding square, together with the vertical and horizontal diameter segments of the disk. For each disk, the resulting arrangement of horizontal/vertical line segments forms an arrangement (a square with a "+" inside it, partitioning the square into four subsquares) of 12 line segments, each of length $r_{i}$. We let $E$ denote this set of $12 n$ line axis-parallel line segments. Let $B B(E)$ denote the axis-aligned bounding box of $E$, which is the same as $B B(\mathcal{D})$, the bounding box of the disks. The edges $E$ have their endpoint coordinates among $\mathcal{I}_{x}$ and $\mathcal{I}_{y}$, and they form a network of horizontal/vertical segments within $B B(E)$, of total length $\lambda(E)=12 \sum_{i} r_{i}$.

We utilize the concept of an " $m$-span" from [22]. Let $\rho$ be an axis-aligned rectangle. The intersection, $\ell \cap(E \cap \rho)$, of a horizontal/vertical cut line $\ell$ with $E \cap \rho$ consists of a discrete (possibly empty) set of line segments and singleton intersection points (which we can consider to be zero-length line segments). Let the endpoints of these segments, be denoted by $q_{1}, \ldots, q_{\xi}$, in order along $\ell$. We define the $m$-span, $\sigma_{m}(\ell)$, of $\ell$ (with respect to $\rho$ ) to be the empty set if $\xi \leq 2(m-1)$, and to be the (possibly zero-length) line segment $q_{m} q_{\xi-m+1}$ otherwise. Refer to Fig. 13 for an example. 
By the $m$-guillotine charging argument 22, we know that the edge set $E$ can be augmented by a set of $m$-span horizontal/vertical segments (bridges), of total length $O(\lambda(E) / m)=O\left((1 / m) \sum_{i} r_{i}\right)$, so that the resulting edge set $E^{\prime}$ is $m$-guillotine with respect to $B B(E)$ in the usual sense defined in [22]: there exists a horizontal/vertical cut for $B B(E)$ such that the $m$-span of $E^{\prime}$ is contained in $E^{\prime}$, and, recursively, the set $E^{\prime}$ is $m$-guillotine with respect to the subrectangles of $\rho$ on either side of the cut. The base case of the recursion is specified by defining $E^{\prime}$ to be $m$-guillotine with respect to rectangle $\rho$ if no vertex (endpoint) of an edge of $E^{\prime}$ lies interior to $\rho$.

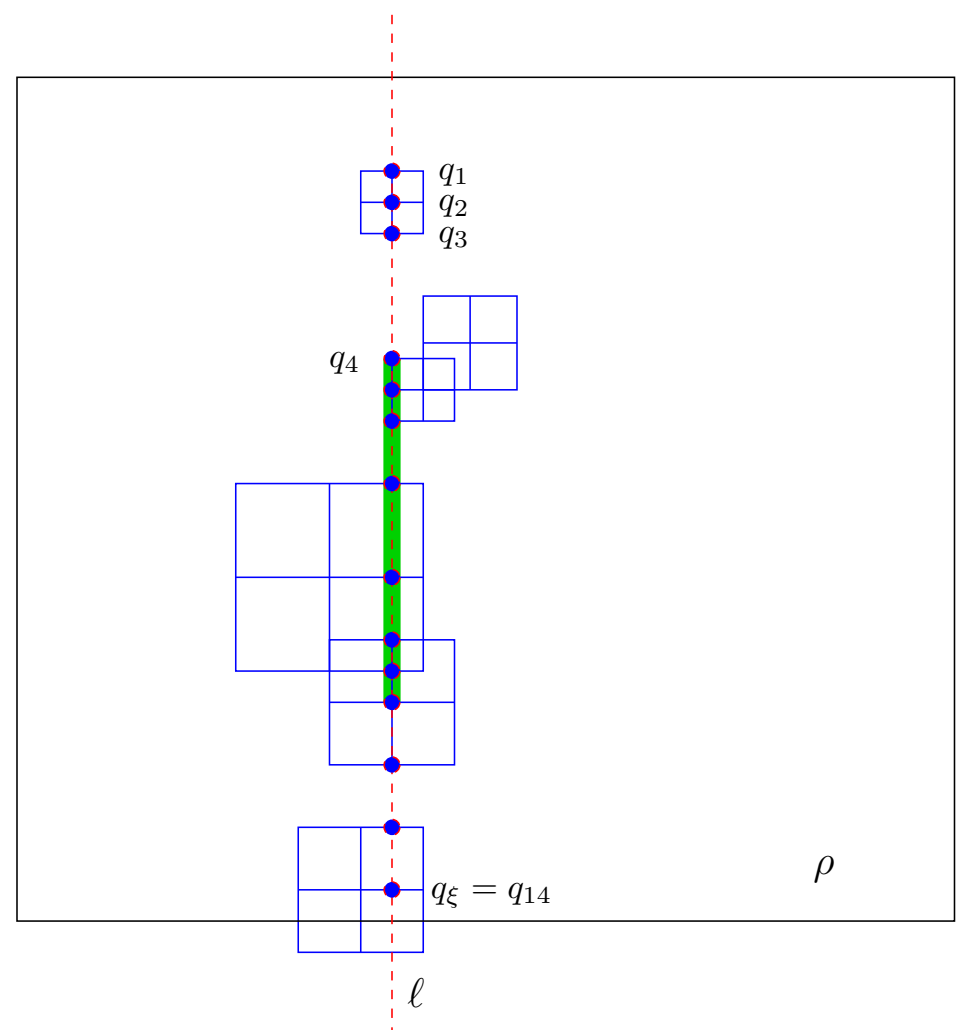

Figure 13: An example of an $m$-span (bold green) for a set of edges $E$, shown in blue. Here, $m=4$, and the cut $\ell$ has $\xi=14$ endpoints of segments of $E$ along it.

We say that an axis-parallel segment $a b \subset \ell$ fully crosses a disk $D$ if $a b$ intersects the interior of $D$ and $a b \cap D=\ell \cap D$; i.e., $a b$ crosses two opposite (top/bottom or left/right) semicircles that comprise the boundary of $D$, so that as one traverses the segment $a b$, one enters $D$ exactly once and then exits $D$ exactly once. Note that if $a b \subset \ell$ fully crosses $D$, then the projection of the center of $D$ onto $\ell$ lies on the segment $a b$. Note too that if segment $a b$ fully crosses a disk $D$, then $a b$ properly crosses the (horizontal/vertical) diameter segment of $D$ that is perpendicular to $a b$

If $E^{\prime}=E$ (i.e., no $m$-span segments had to be added to $E$, as $E$ is already $m$-guillotine as an edge set), then the disks $\mathcal{D}$ are already $m$-guillotine, since the recursive cutting that exhibits that $E$ is $m$-guillotine as a network also serves to establish that the set $\mathcal{D}$ of disks is $m$-guillotine as a set of disks. Specifically, if the $m$-span along a cut $\ell$ of a rectangle $\rho$ is contained in the edge set $E$, then there are at most $O(m)$ points within $\rho$ where $\ell$ crosses edges of $E$, and, in particular, at most $O(m)$ points where $\ell$ properly crosses diameter segments of disks. This implies that the segment $\ell \cap \rho$ fully crosses at most $O(m)$ disks, implying that $\ell \cap \rho$ intersects $O(m)$ disks (Lemma 12 implies that each endpoint of $\ell \cap \rho$ intersects at most 6 disks), making $\ell$ an $m$-perfect cut (for appropriate choices of constants $c_{1}$ and $c_{2}$ ). 
If $E^{\prime} \neq E$, then consider an $m$-span segment, $a b$, that was added to $E$ along some cut $\ell$ in the recursive partitioning of $\rho$. Let $a^{\prime} b^{\prime}=\ell \cap \rho$, with $a$ the endpoint of $a b$ that is closest to $a^{\prime}$ (i.e., the order along $\ell$ is $\left.\left(a^{\prime}, a, b, b^{\prime}\right)\right)$. We then distinguish two cases:

(a) If the $m$-span $a b$ does not fully cross a disk of $\mathcal{D}$, then we know that $a^{\prime} b^{\prime}$ intersects only $O(m)$ disks of $\mathcal{D}$, by the following reasoning. By the definition of $m$-span for $E$, the segments $a^{\prime} a$ and $b b^{\prime}$ each have at most $m$ points along them where the segment crosses (orthogonally) an edge of $E$ corresponding to a diameter segment of a disk; thus, each of $a^{\prime} a$ and $b b^{\prime}$ fully cross at most $m$ disks of $\mathcal{D}$. Since we are assuming that $s b$ does not fully cross any disk, we know that the total number of disks that are fully crossed by $a^{\prime} b^{\prime}$ is $O(m)$. Points $a^{\prime}$ and $b^{\prime}$ each lie within at most 6 disks, by Lemma 12 . Any disk that intersects $a^{\prime} b^{\prime}$ must either contain $a^{\prime}$ or $b^{\prime}$ or be fully crossed by $a^{\prime} b^{\prime}$ (meaning that, when going from $a^{\prime}$ to $b^{\prime}$ along the segment, we both enter and leave the disk). Thus, in total there are $O(m)$ disks of $\mathcal{D}$ that are intersected by $a^{\prime} b^{\prime}=\ell \cap \rho$, implying that $\ell$ is $m$-perfect with respect to $\mathcal{D}$ and $\rho$.

(b) If the $m$-span $a b$ does fully cross at least one disk, then such a disk has the property that the projection of its center point onto $\ell$ lies on the segment $a b$. Assume, without loss of generality, that $\ell$ (and thus $a b)$ is vertical. Then, among disks that $a b$ fully crosses, if there are any with center point to the left of $\ell$, let $D_{L}$ (centered at $p_{L}$, with radius $r_{L}$ ) be a disk with leftmost center point $p_{L}$. Similarly, if there are fully crossed disks with center point to the right of $\ell$, let $D_{R}$ (centered at $p_{R}$, with radius $r_{R}$ ) be a disk with rightmost center point $p_{R}$.

Claim 1. If there are any disks of $\mathcal{D}$ that are fully crossed by ab and have center point to the left of $a b$, then the disk of radius $r_{L}+2|a b|$ centered at $p_{L}$ covers any disk $D \in \mathcal{D}$, with center $p$ and radius $r$, that is fully crossed by $a b$ and has $p$ to the left of ab. Similarly, if there are any disks of $\mathcal{D}$ that are fully crossed by $a b$ and have center point to the right of $a b$, then the disk of radius $r_{R}+2|a b|$ centered at $p_{R}$ covers any disk of $\mathcal{D}$ that is fully crossed by ab and has its center to the right of ab.

Proof. Assume that there is at least one disk of $\mathcal{D}$ that is fully crossed by $a b$ and has its center to the left of $a b$. Then, we let disk $D_{L}$, with center $p_{L}$ and radius $r_{L}$, be one such disk whose center point is leftmost. We know that the center point $p_{L}$ projects onto $a b$; let $h_{L}$ be the distance from $p_{L}$ to $a b$ (i.e., the (horizontal) distance from $p_{L}$ to its projection on the line $\ell$ ). Let $D \in \mathcal{D}$ be a disk, with center $p$ and radius $r$, that is fully crossed by $a b$, with center point $p$ to the left of $a b$, and let $h$ be the (horizontal) distance from $p$ to $a b$. By our choice of $D_{L}$, we know that $h_{L} \geq h$. Let $q \in D$ be any point in disk $D$. Refer to Fig. 14 Then, by the triangle inequality,

$$
\left|p_{L} q\right| \leq\left|p_{L} p\right|+|p q| \text {. }
$$

Also by the triangle inequality, since the points $p_{L}$ and $p$ project onto $a b$ at two points whose $y$ coordinates differ by at most $|a b|$, we get

$$
\left|p_{L} p\right| \leq\left|x\left(p_{L}\right)-x(p)\right|+\left|y\left(p_{L}\right)-y(p)\right| \leq\left(h_{L}-h\right)+|a b|,
$$

where $x(\cdot)$ and $y(\cdot)$ denote $x$ - and $y$-coordinates. Thus, using the fact that $|p q| \leq r$ (since $q \in D$ ), we get that

$$
\left|p_{L} q\right| \leq\left|p_{L} p\right|+|p q| \leq\left(h_{L}-h\right)+|a b|+r .
$$

Again using the triangle inequality, we know that $|p a| \leq h+|a b|$; thus, $h \geq|p a|-|a b|$. Since disk $D$ is fully crossed by $a b$, so that $a$ is not contained in $D$, we know that $|p a| \geq r$, implying that

$$
r-h \leq r-(|p a|-|a b|) \leq|a b| .
$$

Putting this in the above inequality, we get

$$
\left|p_{L} q\right| \leq\left(h_{L}-h\right)+|a b|+r \leq h_{L}+|a b|+|a b| \leq r_{L}+2|a b|
$$

implying that any $q \in D$ is at distance at most $r_{L}+2|a b|$ from the center point $p_{L}$, as we claimed.

The proof for disks with center points to the right of $a b$ is symmetric. 


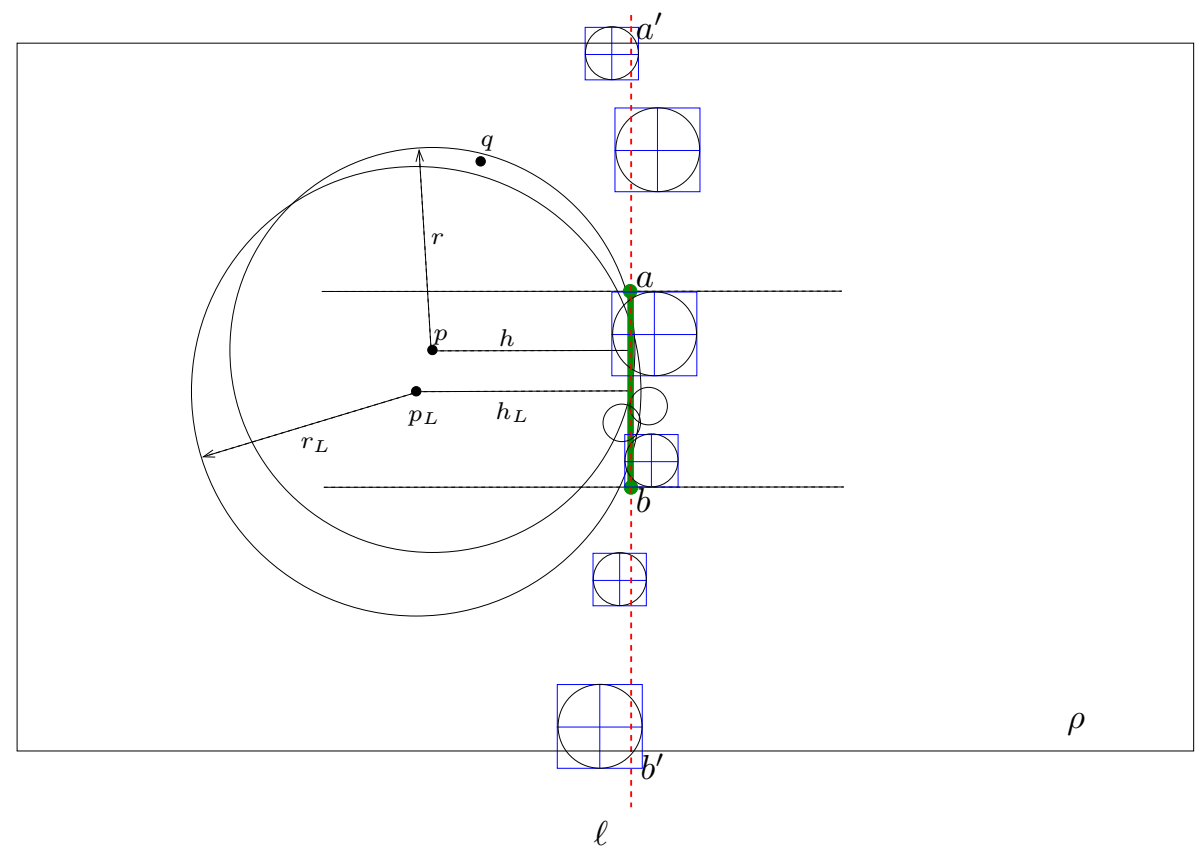

Figure 14: Illustration of the proof of Claim 1. The vertical cut $\ell$ intersects the rectangle $\rho$ in the segment $a^{\prime} b^{\prime}$. The $m$-span $(m=4), a b$, is shown in thick green. The disk $D_{L}$, centered at $p_{L}$, of radius $r_{L}$, is shown, as is another disk $D$, centered at $p$, with radius $r$. The claim is that any point $q \in D$ must be covered by an enlarged disk, of radius $r_{L}+2|a b|$, centered on $p_{L}$.

We now modify the set $\mathcal{D}$ of disks as follows. If there are disks fully crossed by $a b$ with center to the left of $a b$, we select one, $D_{L}$, having leftmost center point, and increase its radius to $r_{L}+2|a b|$. By Claim 1. we know that this new enlarged disk covers all disks $D_{i} \in \mathcal{D}$ that were fully crossed by $a b$, with center points $p_{i}$ to the left of $a b$. This implies that we can remove all such disks $D_{i}$ (i.e., shrink $r_{i}$ to zero), while maintaining connectivity of the set of disks. If there are disks fully crossed by $a b$ whose center points are to the right of $a b$, we similarly enlarge one of them, $D_{R}$, whose center point $p_{R}$ is rightmost, while removing the others (shrinking their radii to zero). The net change to the sum of the radii of all disks is that it goes up by at most $O(|a b|)$.

As in case (a) above, we know that the segments $a^{\prime} a$ and $b b^{\prime}$ each intersect $O(m)$ disks. After the modification above, we know that $a b$ intersects at most $O(1)$ disks (at most 2 (enlarged) disks of radii $r_{L}+2|a b|$ and $r_{R}+2|a b|$, and at most 6 disks containing $a$ and 6 disks containing $b$ ). Thus, in total $a^{\prime} b^{\prime}=\ell \cap \rho$ intersects at most $O(m)$ disks of $\mathcal{D}$, so we know that $\ell$ is $m$-perfect with respect to $\mathcal{D}$ and $\rho$.

Applying the above modification of the disk radii to all cuts in the recursive set of cuts associated with the $m$-guillotine edge set $E^{\prime}$, we obtain a set of disks $\mathcal{D}_{m}$. Since the sum of all $m$-span lengths $|a b|$ is charged off to the length $\lambda(E)=O\left(\sum_{i} r_{i}\right)$ of the edge set $E$, totalling at most $O(1 / m)$ times the sum $\sum_{i} r_{i}$, we have shown that the sum of the radii of disks of $\mathcal{D}_{m}$ is at most $(1+(C / m)) \sum_{i} r_{i}$.

Finally, by Lemma 11, we can round up the radii of the resulting disks $\mathcal{D}_{m}$ to make them $\mathcal{R}$-disks, while increasing the sum of radii by at most a factor $1+O(1 / m)$.

\subsection{Dynamic Programming}

We now give an algorithm to compute a minimum-cost (sum of radii) $m$-guillotine set of $\mathcal{R}$-disks whose union is connected. The algorithm is based on dynamic programming. A subproblem is specified by a rectangle, $\rho$, with $x$ - and $y$-coordinates among the sets $\mathcal{I}_{x}$ and $\mathcal{I}_{y}$, respectively, of discrete coordinates. The 
subproblem includes specification of boundary information, for each of the four sides of $\rho$. Specifically, the boundary information includes:

(i) $O(m)$ "portal disks", which are $\mathcal{R}$-disks intersecting the boundary, $\partial \rho$, of $\rho$, with at most $O(m)$ disks specified per side of $\rho$; and,

(ii) a connection pattern, specifying which subsets of the portal disks are required to be connected within $\rho$. (Thus, the connection pattern is specified by giving a partition of the set of portal disks; while the number of partitions is exponential in $m$, it is constant for fixed $\epsilon=\lceil 2 / m\rceil$.)

There are a polynomial number of subproblems (specifically, $n^{O(m)}$ ), for any fixed $m$. For a given subproblem, the dynamic program optimizes over all (polynomial number of) possible cuts $\ell$ (horizontal at $\mathcal{I}_{y}$-coordinates or vertical at $\mathcal{I}_{x}$-coordinates), and choices of up to $O(m) \mathcal{R}$-disks intersecting the cut segment $\ell \cap \rho$, along with all possible compatible connection patterns for each side of the cut. (The optimal value of the objective function for each of the two corresponding subproblems on each side of each candidate cut $\ell$ have been precomputed and tabulated, since we fill in the table of data in order of increasing (combinatorial) size of the rectangles $\rho$.) The result is an optimal $m$-guillotine set of $\mathcal{R}$-disks such that their union is connected and the sum of the radii is minimum possible for $m$-guillotine sets of $\mathcal{R}$-disks. Since we know, from the structure theorem, that an optimal set of disks centered at points $P$ can be converted into an $m$-guillotine set of $\mathcal{R}$-disks centered at points of $P$, whose union is connected, and we have computed an optimal such structure, we know that the disks obtained by our dynamic programming algorithm yield an approximation to an optimal set of disks. In summary, we have shown the following result:

Theorem 14. For any $\epsilon>0$, there is an approximation algorithm for CRA running in time $n^{O(1 / \epsilon)}$ that produces an approximate solution within factor $(1+\epsilon)$ of the optimal.

\section{Experimental Results}

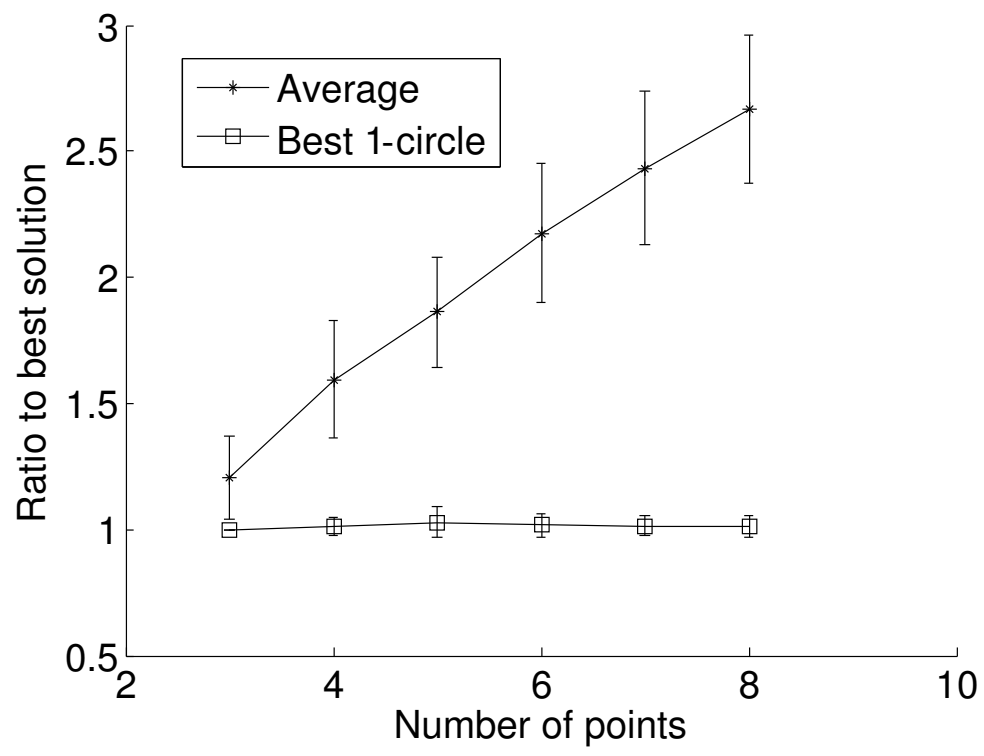

Figure 15: Ratios of the average over all enumerated trees and of the best 1-disk tree to the optimal $\sum r_{i}$. Results were averaged over 100 trials for each number.

It is interesting that even in the worst case, a one-disk solution is close to being optimal. This is supported by experimental evidence. Using the MATLAB programming language, we analyzed the difficulty associated 
with finding the optimal assignment of radii in randomized trials. In order to generate random problem instances, we considered different numbers of points, uniformly distributed in a two-dimensional circular region. For each trial considering a single distribution of points, we enumerated all possible spanning trees using the method described in [3] and recorded the optimal value with the algorithm described in Section 2. Additionally, for each trial, we noted the sum of $R$ values obtained from the best one-disk solution. Fig. 15 shows that the ratio of the best one-disk solution to the best solution remains relatively flat as the number of points increases, as opposed to the average solution, suggesting that the one-disk solution is an excellent heuristic choice. These results were obtained in less than 6 hours using an i7 PC.

\section{Conclusion}

A number of open problems remain. One of the most puzzling is the issue of complexity in the absence of upper bounds on the radii. The strong performance of the one-disk solution (and even better of solutions with a higher, but limited, number of disks), and the difficulty of constructing solutions for which the one-disk solution is not optimal strongly hint at the possibility of the problem being polynomially solvable. Another indication is that our positive results for one or two disks only needed triangle inequality, i.e., they did not explicitly make use of geometry.

One possible approach to this problem may be to use methods from linear programming. Modeling the objective function and the variables is straightforward; describing the connectivity of a spanning tree by linear cut constraints is also well known. However, even though separating over the exponentially many cut constraints is polynomially solvable (and hence optimizing over the resulting polytope), the overall polytope is not necessarily integral. On the other hand, we have been unable to prove NP-hardness without upper bounds on the radii, even in the more controlled context of graph-induced distances. Note that some results were obtained by means of linear programming: the tight lower bound for 2-disk solutions (shown in Fig. 11) was found by solving appropriate LPs.

Other open problems are concerned with the worst-case performance of heuristics using a bounded number of disks. We showed that two disks suffice for a $\frac{4}{3}$-approximation in general, and a $\frac{5}{4}$-approximation on a line; we conjecture that the general performance guarantee can be improved to $\frac{5}{4}$, matching the existing lower bound. Obviously, the same can be studied for $k$ disks, for any fixed $k$; at this point, the best lower bounds we have are $\frac{7}{6}$ for $k=3$ and $1+\frac{1}{2^{k+1}}$ for general $k$. We also conjecture that the worst-case ratio $f(k)$ of a best $k$-disk solution approximates the optimal value arbitrarily well for large $k$, i.e., $\lim _{k \rightarrow \infty} f(k)=1$.

We have given a PTAS for CRA in two dimensions with unbounded radii, a problem with unknown complexity - there might be a polynomial-time exact solution. Does there exist a PTAS for the general case of CRA with bounded radii, a problem we have shown to be NP-hard? In the conference version of the paper [12, we sketched an approach for how to modify our PTAS for unbounded radii to address a special case of the CRA problem with bounded radii, if an additional assumption is made, that for any segment $p q$, with $p$ and $q$ within feasible disks, there exists a (connected) path of feasible disks whose sum of radii is $O(|p q|)$. It would be interesting to obtain a PTAS for the CRA for the general case of bounded radii.

\section{Acknowledgments}

We thank Ferran for being a great inspiration to all of us. A preliminary version of this work appears in the Algorithms and Data Structures Symposium (WADS), 2011 [12. This work was started during the 2009 McGill/INRIA/University of Victoria Bellairs Workshop on Computational Geometry. We thank all other participants for contributing to the great atmosphere. This work has been partially supported by the National Science Foundation (grants CCF-1054779 and IIS-1319573, Erin Chambers; grants CCF1018388 and CCF-1526406, Joseph Mitchell), by the Binational Science Foundation (BSF 2010074, Joseph Mitchell), Sandia National Labs (Joseph Mitchell), and three individual NSERC Discovery grants (one each for Venkatesh Srinivasan, Ulrike Stege, and Sue Whitesides). 


\section{References}

\section{References}

[1] M. Abellanas, A. García Olaverri, F. Hurtado, J. Tejel, and J. Urrutia. Augmenting the connectivity of geometric graphs. Comput. Geom., 40(3):220-230, 2008.

[2] H. Alt, E. M. Arkin, H. Brönnimann, J. Erickson, S. P. Fekete, C. Knauer, J. Lenchner, J. S. B. Mitchell, and K. Whittlesey. Minimum-cost coverage of point sets by disks. In Proc. 22nd ACM Symp. Comp. Geom. (SoCG), pages 449-458, 2006.

[3] D. Avis and K. Fukuda. Reverse search for enumeration. Disc. Appl. Math., 65(1-3):21-46, 1996.

[4] C. Baur and S. P. Fekete. Approximation of geometric dispersion problems. Algorithmica, 30(3):451-470, 2001.

[5] V. Bilò, I. Caragiannis, C. Kaklamanis, and P. Kanellopoulos. Geometric clustering to minimize the sum of cluster sizes. In In Proc. 13th European Symp. Algorithms, Vol 3669 of LNCS, pages 460-471, 2005.

[6] G. Calinescu, I. I. Mandoiu, P.-J. Wan, and A. Zelikovsky. Selecting forwarding neighbors in wireless ad hoc networks. Mobile Networks and Applications, 9:101-111, 2004.

[7] G. Calinescu and P. J. Wan. Range assignment for high connectivity in wireless ad hoc networks. In Proc. 2nd International Conference on AD-HOC Networks and Wireless (AdHoc-NOW'03), pages $235-246,2003$.

[8] I. Caragiannis, C. Kaklamanis, and P. Kanellopoulus. Energy-efficient wireless network design. Theory of Computing Systems, 39:593-617, 2006.

[9] P. Carmi and M. J. Katz. Power assignment in radio networks with two power levels. In Proc. 9th Scandinavian Workshop on Algorithm Theory, pages 431-441, 2004.

[10] P. Carmi, M. J. Katz, and J. S. B. Mitchell. The minimum-area spanning tree problem. Comput. Geom. Theory Appl., 35:218-225, October 2006.

[11] P. Carmi, M. J. Katz, M. Segal, and H. Shpungin. Fault-tolerant power assignment and backbone in wireless networks. In Proc. IEEE Internat. Workshop on Foundations and Algorithms for Wireless Networking, pages 80-84, 2006.

[12] E. Chambers, S. P. Fekete, H.-F. Hoffman, D. Marinakis, J. S. B. Mitchell, V. Srinivasan, U. Stege, and S. Whitesides. Connecting a set of circles with minimum sum of radii. In Algorithms and Data Structures- 12th International Symposium (WADS2001), number 6844 in Springer LNCS, pages 183$194,2011$.

[13] M. Charikar and R. Panigrahy. Clustering to minimize the sum of cluster diameters. J. Comput. Syst. Sci., 68:417-441, March 2004.

[14] A. E. Clementi, P. Penna, and R. Silvestri. On the power assignment problem in radio networks. Mobile Networks and Applications, 9(2):125-140, 2004.

[15] S. Doddi, M. V. Marathe, S. S. Ravi, D. S. Taylor, and P. Widmayer. Approximation algorithms for clustering to minimize the sum of diameters. Nordic J. of Computing, 7:185-203, September 2000.

[16] B. Fuchs. On the hardness of range assignment problems. Networks, 52(4):183-195, 2008.

[17] M. Gibson, G. Kanade, E. Krohn, I. A. Pirwani, and K. Varadarajan. On clustering to minimize the sum of radii. In Proc. 19th ACM-SIAM Symp. Disc. Alg. (SODA), pages 819-825, 2008. 
[18] N. Lev-Tov and D. Peleg. Polynomial time approximation schemes for base station coverage with minimum total radii. Computer Networks, 47(4):489-501, 2005.

[19] D. Lichtenstein. Planar formulae and their uses. SIAM J. Comput., 11(2):329-343, 1982.

[20] E. L. Lloyd, R. Liu, M. V. Marathe, R. Ramanathan, and S. S. Ravi. Algorithmic aspects of topology control problems for ad hoc networks. Mobile Networks and Applications, 10:19-34, 2005.

[21] D. Marinakis, D. Meger, I. Rekleitis, and G. Dudek. Hybrid inference for sensor network localization using a mobile robot. In AAAI National Conference on Artificial Intelligence, pages 1089-1094, Vancouver, Canada, July 2007.

[22] J. S. B. Mitchell. Guillotine subdivisions approximate polygonal subdivisions: A simple polynomialtime approximation scheme for geometric TSP, $k$-MST, and related problems. SIAM J. Comput., 28(4):1298-1309, 1999.

[23] P.-J. Wan, G. Calinescu, X.-Y. Li, and O. Frieder. Minimum-energy broadcast routing in static ad hoc wireless networks. ACM Wireless Networks, 8:607-617, 2002. 